\title{
Rapid evaluation of two-dimensional retarded time integrals
}

\author{
D. A. Bizzozero, J. A. Ellison, K. A. Heinemann, and S. R. Lau ${ }^{1}$ \\ Mathematics and Statistics, University of New Mexico, Albuquerque, NM 87131
}

January 4, 2017

\begin{abstract}
We present two methods for rapid evaluation of two-dimensional retarded time integrals. For example, such integrals arise as the $z=0$ trace $U(t, x, y, 0)$ of a solution $U(t, x, y, z)$ to $3+1$ wave equation $\square U=$ $-2 f(t, x, y) \delta(z)$ forced by a "sheet source" at $z=0$. The spatial Fourier transform of a two-dimensional retarded time integral involves a temporal convolution with the zeroth order Bessel function $J_{0}(t)$. Appealing to work by Alpert, Greengard, and Hagstrom and by Xu and Jiang on rational approximation in the Laplace-transform domain, our first method relies on approximation of $J_{0}(t)$ as a sum of exponential functions. We achieve approximations with double precision accuracy near $t \simeq 0$, and maintain single precision accuracy out to $T \simeq 10^{8}$. Our second method involves evolution of the $3+1$ wave equation in a "thin block" above the sheet, adopting the radiation boundary conditions of Hagstrom, Warburton, and Givoli based on complete plane wave expansions. We review their technique, present its implementation for our problem, and present new results on the nonlocal spacetime form of radiation boundary conditions. Our methods are relevant for the sheet-bunch formulation of the Vlasov-Maxwell system, although here we only test methods on a model problem, a Gaussian source following an elliptical orbit. Our concluding section discusses the complexity of both methods in comparison with naive evaluation of a retarded-time integral.
\end{abstract}

Keywords: retarded time integral, rational approximation, radiation boundary conditions, initial boundary value problem, Vlasov-Maxwell system, accelerator beam physics.

2010 MSC: 35L05, 41A20, 65A05, 65M99, 65Z05, 78M25

\section{INTRODUCTION AND PRELIMINARIES}

1.1. Introduction. This article presents two strategies for fast numerical evaluation of the retarded-time integral

$$
F(t, \mathbf{x}):=\frac{1}{2 \pi} \int_{\left|\mathbf{x}-\mathbf{x}^{\prime}\right| \leq t} d \mathbf{x}^{\prime} \frac{f\left(t-\left|\mathbf{x}-\mathbf{x}^{\prime}\right|, \mathbf{x}^{\prime}\right)}{\left|\mathbf{x}-\mathbf{x}^{\prime}\right|},
$$

where $\mathbf{x}=(x, y), d \mathbf{x}^{\prime}=d x^{\prime} d y^{\prime}$, and we have included the $(2 \pi)^{-1}$ in (1) for later convenience. Both strategies are useful when the integral must be evaluated over a range of spatial $\mathbf{x}$ and temporal $t$ points. Of particular interest is the support of $F(t, \cdot)$ in the case when the support of $f(t, \cdot)$ is small for all past times. One scenario involving integrals of this form is the Maxwell equations with charge and current densities that are confined to a "sheet" $z=0$ and have small supports. For this scenario, the $z=0$ restrictions of the electric field $E_{x}$, $E_{y}$ and the magnetic field $B_{z}$ components obey (1), in each case with $f(t, \mathbf{x})$ appropriately

\footnotetext{
${ }^{1}$ Corresponding author, lau@math.unm.edu
} 
determined by the sheet charge or sheet current densities. The $z=0$ restrictions of the remaining components $E_{z}, B_{x}, B_{y}$ vanish.

This scenario arises in a "sheet bunch model" of the Vlasov-Maxwell system describing an electron bunch in the bunch compressor of a free electron laser, for example, the LCLS at Stanford, CA. For more details on the sheet bunch model, see [1, 2, 3, 4]. Indeed, in this model the charge and current densities of an electron bunch are confined to the "sheet" $z=0$, since they correspond to a phase space density obeying the Vlasov equation and confined to $z=0, p_{z}=0$. Here, $p_{z}$ is the $z$-component of the electron momentum. Since the time evolutions of the charge and current densities are governed by the Vlasov equation, which is in turn determined by the Lorentz force in the sheet, $E_{x}, E_{y}$ and $B_{z}$ are the only electromagnetic fields which act on the source. Whence for this scenario only (1) matters. Of course, a complete Vlasov-Maxwell treatment would involve charge/current densities which are not confined to $z=0$. The high cost involved in numerically modelling the full 3D problem motivates an approximation like the 2D sheet bunch or a 1D treatment based on line charges. The need to rapidly evaluate (1) has arisen in our work on the sheet bunch model. Indeed, retarded-time integration is the bottleneck in the sheet-bunch modelling code VM3@A (VlasovMaxwell Monte-Carlo Method at Albuquerque). In that context straightforward integration is slow, since for each $t$ the integral must be recomputed from scratch, i.e. knowledge of $F(t, \mathbf{x})$ is not reused in the computation of $F(t+\Delta t, \mathbf{x})$. This issue is more evident in another expression (2) for the integral (1) given below. Moreover, straightforward integration is memory intensive as the time history of $f(t, \mathbf{x})$ must be stored.

The outline of this article is as follows. The next subsection collects some background material. Section 2 describes our first approach based on Fourier transformation and approximation of the Bessel function $J_{0}(z)$ as a sum of exponentials. Section 3 describes our second approach based on evolution of the ordinary wave equation with appropriate radiation boundary conditions. Both of these sections present numerical results (cf. Subsections 2.2 and 3.4) based on the following model. We choose $f(t, \mathbf{x})$ as a narrow Gaussian which moves subluminally along an elliptical trajectory; see Eq. (14) below. Section 3 is more involved theoretically, and it presents some new results concerning radiation boundary conditions [5, 6] developed by Hagstrom, Warburton, and Givoli. The concluding Section 4 summarizes our findings and compares our two approaches from the standpoint of our target application. Appendices focus on several intricacies overlooked in the main sections. In particular, Appendices $\mathrm{A}$ and $\mathrm{B}$ describe numerical tables approximating $J_{0}(t)$ as a sum of exponential and how such tables are constructed. Here we rely on our own modified version of the Alpert-Greengard-Hagstrom "compression algorithm" [7] for rational approximation, with important additional input from the work $[9,10]$ of Jiang and $\mathrm{Xu}$ and Jiang. Beylkin and Monzon also describe a procedure [8] for approximation of $J_{0}(t)$ and other functions by exponential sums. When represented in double precision format, one of our approximations (comprised of 150 terms) maintains a $10^{-14}$ relative accuracy for $0 \leq t \leq 300$ and a $10^{-10}$ relative accuracy for $0 \leq t \leq 10^{6}$. When represented in quadruple precision format, the same approximation maintains a $10^{-15}$ relative accuracy for $0 \leq t \leq 10^{8}$. Appendix $\mathrm{C}$ establishes our main result on the spacetime form of the relevant exact radiation boundary conditions.

1.2. Preliminaries. Here we collect the theoretical background underlying our two methods for fast evaluation of (1), showing that spatial Fourier transformation of (1) yields a temporal convolution involving the Bessel function $J_{0}(t)$ (the background for Method 1 ) and describing 
the relationship between the two-dimensional integral (1) and solutions to the ordinary $3+1$ (three-space plus time) wave equation (the background for Method 2). In the following we assume that $f$ has the smoothness properties needed to justify our calculations.

1.2.1. Analytical background. We first describe the background for Method 1. Through the change of variables $x^{\prime}=x+(t-\tau) \cos \phi$ and $y^{\prime}=y+(t-\tau) \sin \phi$, Eq. (1) becomes

$$
F(t, \mathbf{x})=\frac{1}{2 \pi} \int_{0}^{t} d \tau \int_{0}^{2 \pi} d \phi f(\tau, \mathbf{x}+(t-\tau) \boldsymbol{\nu}(\phi)),
$$

where $\boldsymbol{\nu}(\phi)=(\cos \phi, \sin \phi)$. As mentioned, (2) shows that the present field $F(t, \cdot)$ is not of direct use in computing the future value $F(t+\Delta t, \mathbf{x})$. To gain insight into (2) and (1), we investigate the support of $F(t, \cdot)$ in terms of the support of $f(t, \cdot)$. In the physical scenario above, $F(t, \cdot)$ corresponds to electromagnetic-field components and $f(t, \cdot)$ to charge and current densities associated with the electron bunch; therefore, we respectively refer to these spatial supports as the field support and the bunch support.

The contribution

$$
g(\tau):=\frac{1}{2 \pi} \int_{0}^{2 \pi} d \phi f(\tau, \mathbf{x}+(t-\tau) \boldsymbol{\nu}(\phi))
$$

at time $\tau$ to $F(t, \mathbf{x})=\int_{0}^{t} d \tau g(\tau)$ is pictured in Fig. 1a. The figure shows the bunch support $\mathcal{S}_{f}(\tau):=\left\{\mathbf{x} \in \mathbb{R}^{2}: f(\tau, \mathbf{x}) \neq 0\right\}$ in the $(x, y)$-plane at time $\tau$. The dashed circle in Fig. 1a is the set $\mathcal{C}_{\mathbf{x}}(t-\tau):=\left\{\mathbf{x}^{\prime} \in \mathbb{R}^{2}:\left|\mathbf{x}-\mathbf{x}^{\prime}\right|=t-\tau\right\}$, i.e. the circle centered at $\mathbf{x}=(x, y)$ and with radius $r=t-\tau$. The integrand in $g(\tau)$ is nonzero if and only if the intersection $\mathcal{C}_{\mathbf{x}}(t-\tau) \cap \mathcal{S}_{f}(\tau)$ is nonempty. That is, a point in the bunch contributes to the $\phi$-integration in $g(\tau)$ if and only if it lies on $\mathcal{C}_{\mathbf{x}}(t-\tau)$. Figure 1a shows the bunch intersecting $\mathcal{C}_{\mathbf{x}}(t-\tau)$; were the bunch off $\mathcal{C}_{\mathbf{x}}(t-\tau)$ there would be no contribution to $g(\tau)$.

The field support $\mathcal{S}_{F}(t):=\left\{\mathbf{x} \in \mathbb{R}^{2}: F(t, \mathbf{x}) \neq 0\right\}$ is also given by

$$
\mathcal{S}_{F}(t)=\left\{\mathcal{C}_{\mathbf{x}}(t-\tau) \cap \mathcal{S}_{f}(\tau): \tau \in[0, t]\right\},
$$

which can be pictured in terms of the backward lightcone emanating from $(t, \mathbf{x})$, as shown in Fig. 1b. The cross section of the cone at arbitrary $\tau \in[0, t]$ is shown as a dashed circle, the same one as in Fig. 1a. Each bunch in the sequence shown in Fig. 1b should be visualized as lying in a fixed constant- $\tau$ spatial plane. We remark on two possible scenarios. First, if $\tau \lesssim t$ and the spatial evaluation point $\mathbf{x}$ for $F(t, \mathbf{x})$ is contained within the bunch support $S_{f}(t)$ at the current time $t$, then $\mathcal{C}_{\mathbf{x}}(t-\tau) \cap S_{f}(\tau)=\mathcal{C}_{\mathbf{x}}(t-\tau)$ (so the corresponding integration in (3) will run over all $\phi$ angles) and, of course, $\mathbf{x} \in S_{F}(t)$. Second, if $t$ is large and $\tau>0$ is small (also with $\mathcal{S}_{f}(\tau)$ compact for all past times and the bunch moving subluminally), then the bunch will lie inside of the past lightcone, $\mathcal{C}_{\mathbf{x}}(t-\tau) \cap S_{f}(\tau)$ will be empty, and $\mathbf{x} \notin S_{F}(t)$.

Fourier transformation gives

$$
\widehat{F}(t, \boldsymbol{\xi}):=\frac{1}{2 \pi} \int_{\mathbb{R}^{2}} d \mathbf{x} e^{-\mathrm{i} \mathbf{x} \cdot \boldsymbol{\xi}} F(t, \mathbf{x})=\int_{0}^{t} d \tau \widehat{f}(\tau, \boldsymbol{\xi})\left(\frac{1}{2 \pi} \int_{0}^{2 \pi} d \phi e^{\mathrm{i}(t-\tau) \boldsymbol{\nu}(\phi) \cdot \boldsymbol{\xi}}\right),
$$

where the first equality establishes our Fourier transform notation. Using a standard integral representation for $J_{0}(t)$, we then find

$$
\widehat{F}(t, \boldsymbol{\xi})=\int_{0}^{t} d \tau \widehat{f}(\tau, \boldsymbol{\xi}) J_{0}(\xi(t-\tau))
$$




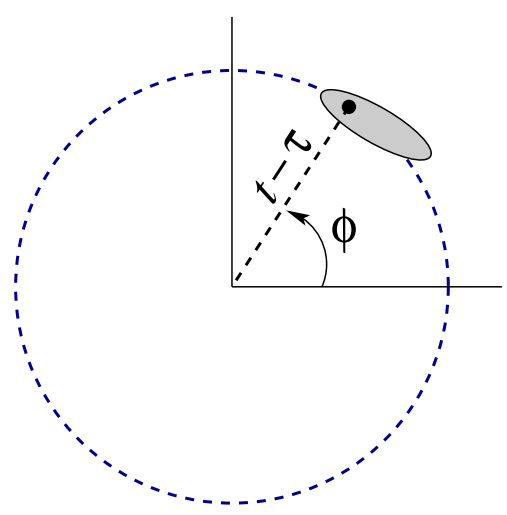

(a) Spatial slice of history.

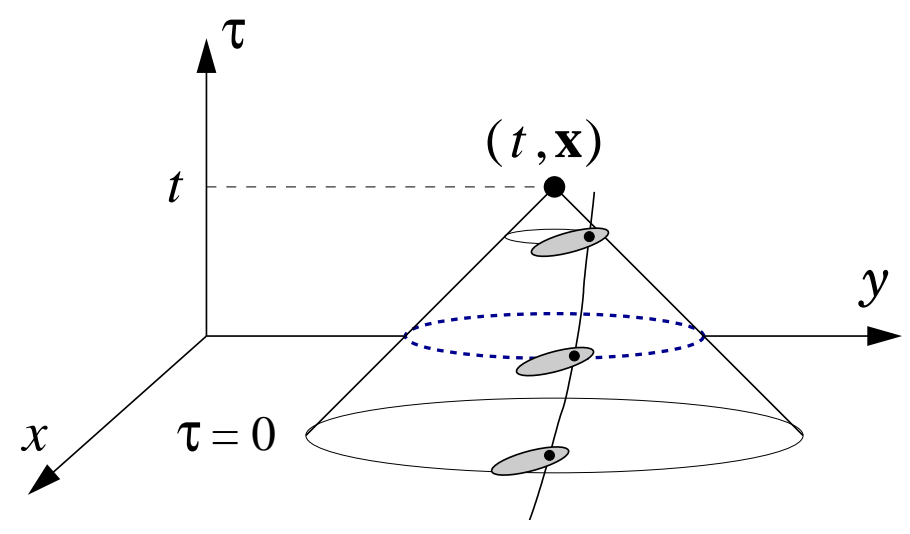

(b) Spacetime history.

Figure 1. Retarded-Time integration in TERMS OF BUNCH HiSTORY. In the right-hand figure the $z$-coordinate normal to the sheet is suppressed.

as the aforementioned convolution. Here $\xi=|\boldsymbol{\xi}|$, and the absence of a numerical factor in front of the integral in (6) is due to the $(2 \pi)^{-1}$ factor chosen in (1). We emphasize that $F(t, \cdot)$ is needed on its full support in order to carry out the Fourier transform. The Laplace transform of (6) plays an important role in both Methods 1 and 2; it reads as follows:

$$
\stackrel{\vee}{F}(s, \boldsymbol{\xi})=\stackrel{\vee}{f}(s, \boldsymbol{\xi})\left(s^{2}+\xi^{2}\right)^{-1 / 2} .
$$

We use the check notation to denote the Fourier-Laplace transform. Inverting, we obtain for $T>0$

$$
F(t, \mathbf{x})=\frac{1}{4 \mathrm{i} \pi^{2}} \int_{\mathbb{R}^{2}} d \boldsymbol{\xi} \int_{T^{-1}-\mathrm{i} \infty}^{T^{-1}+\mathrm{i} \infty} d s e^{s t+\mathrm{i} \boldsymbol{\xi} \cdot \mathbf{x}} \frac{\stackrel{\vee}{f}(s, \boldsymbol{\xi})}{\sqrt{s^{2}+\xi^{2}}}
$$

Our approximations require $T \gg 1$, i.e. our Bromwich contour is close to the imaginary axis. Finally, we note that (6) appears as Eq. (2.13) in Ref. [11] which considered the inverse problem from our own: recovery of $f$ from $F$ given as data.

We now turn to the background for Method 2. Eq. (1) stems from the following sheetsource IVP for $U=U(t, \mathbf{X})$ :

$$
\begin{array}{r}
\square U=-2 f(t, x, y) \delta(z), \quad t>0, \mathbf{X} \in \mathbb{R}^{3} \\
U=0=\partial_{t} U, \quad t=0, \mathbf{X} \in \mathbb{R}^{3},
\end{array}
$$

where $\mathbf{X}=(\mathbf{x}, z)=(x, y, z)$. The Fourier-Laplace transform (in $\mathbf{x}$ and $t$ ) of the solution is

$$
\stackrel{v}{U}(s, \boldsymbol{\xi}, z)=\frac{\stackrel{v}{f}(s, \boldsymbol{\xi})}{\sqrt{s^{2}+\xi^{2}}} \exp (-\gamma|z|), \quad \gamma=\sqrt{s^{2}+\xi^{2}} .
$$

Comparison with (7) determines that $F$ from (1) and $U$ from (9) are related by

$$
F(t, \mathbf{x})=U(t, \mathbf{x}, 0) .
$$


For $z \geq 0$ the IVP (9) is equivalent to the following initial boundary value problem (IBVP):

$$
\begin{array}{r}
\square U=0, \quad t>0, \mathbf{X} \in \mathbb{R}_{+}^{3} \\
U=0=\partial_{t} U, \quad t=0, \mathbf{X} \in \mathbb{R}_{+}^{3} \\
\partial_{z} U=-f, \quad t>0, \mathbf{X} \in \partial \mathbb{R}_{+}^{3},
\end{array}
$$

referred to here as the open problem on the half space $\mathbb{R}_{+}^{3}=\left\{\mathbf{X}=(\mathbf{x}, z): \mathbf{x} \in \mathbb{R}^{2}, z>0\right\}$ with boundary (the sheet) $\partial \mathbb{R}_{+}^{3}=\left\{\mathbf{X}=(\mathbf{x}, z): \mathbf{x} \in \mathbb{R}^{2}, z=0\right\}$. Method 2 is based on this IBVP, and through the inverse Fourier-Laplace transform we have (again, $z \geq 0$ )

$$
U(t, \mathbf{x}, z)=\frac{1}{4 \mathrm{i} \pi^{2}} \int_{\mathbb{R}^{2}} d \boldsymbol{\xi} \int_{T^{-1}-\mathrm{i} \infty}^{T^{-1}+\mathrm{i} \infty} d s e^{s t+\mathrm{i} \boldsymbol{\xi} \cdot \mathbf{x}-\left(s^{2}+\xi^{2}\right)^{1 / 2} z} \frac{\stackrel{v}{f}(s, \boldsymbol{\xi})}{\sqrt{s^{2}+\xi^{2}}} .
$$

Note that (11) also holds for $F$ from (1) and $U$ from (12).

1.2.2. Numerical background. Numerical results reported below rely on the specific source function (its spatial Fourier transform is given for later reference)

$$
f(t, \mathbf{x})=\theta(t) \exp \left(-\nu\left|\mathbf{x}-\mathbf{x}_{c}(t)\right|^{2}\right), \quad \widehat{f}(t, \boldsymbol{\xi})=\frac{1}{2} \theta(t) \nu^{-1} \exp \left(-\mathrm{i} \mathbf{x}_{c}(t) \cdot \boldsymbol{\xi}\right) \exp \left(-\frac{1}{4} \nu^{-1} \xi^{2}\right)
$$

with $\mathbf{x}_{c}(t)=\left(\lambda_{x} \cos (\omega t), \lambda_{y} \sin (\omega t)\right)$ and the parameters $\lambda_{x}=0.8, \lambda_{y}=1.2, \nu=5.0$, and $\omega=0.8$. Included in the expression (14) for the source is a masking function,

$$
\theta(t)= \begin{cases}0 & \text { for } t \leq 0 \\ \frac{1}{2} \operatorname{erf}\left(\sqrt{\sigma}\left(t-\frac{1}{2} \vartheta\right)\right)+\frac{1}{2} & \text { for } t \in(0, \vartheta) \\ 1 & \text { for } t \geq \vartheta\end{cases}
$$

which ensures that the source is "turned on" gradually. For the results reported below $\vartheta=1.25$ and $\sigma=100$. The simple source (14) models a sheet bunch of electrons clustered as a Gaussian moving on an elliptical orbit. With the parameter choices above, the central velocity $\mathbf{x}_{c}^{\prime}(t)$ of the source does not exceed the unit wave speed, and for all $t$ the source $f(t, \mathbf{x})$ is localized in a small region. As the $(x, y)$-domain we take a rectangle $R=\left[x_{\min }, x_{\max }\right] \times$ $\left[y_{\min }, y_{\max }\right]$ which contains the elliptical orbit of the source from $t=0$ up to a final time $t_{\mathrm{f}}$. Moreover, we must choose $R$ large enough to ensure that it contains the effective support of $F\left(t_{\mathrm{f}}, \cdot\right)$. Since we have chosen a Gaussian "bunch" (not of compact support), the discussion in Subsection 1.2 should be modified to include the notion of effective support. For example, we could introduce the bunch $\epsilon$-support $\mathcal{S}_{f}^{\epsilon}(t):=\left\{\mathbf{x} \in \mathbb{R}^{2}:|f(t, \mathbf{x})|>\epsilon\right\}$, with, say, $\epsilon$ as machine precision. Likewise, we could introduce the field $\epsilon$-support $\mathcal{S}_{F}^{\epsilon}(t)$. Without giving a precise formulation, we note that $R$ must be large enough to ensure that the boundary $\partial R$ is causally disconnected from $\mathcal{S}_{f}^{\epsilon}(t)$ for $t \in\left[0, t_{\mathrm{f}}\right]$. With this condition, $R$ contains $\mathcal{S}_{F}^{\epsilon \cdot t_{\mathrm{f}}}\left(t_{\mathrm{f}}\right)$.

Each of our two methods produces nodal values $F_{k \ell}(t) \simeq F\left(t, x_{k}, y_{\ell}\right)$ which approximate (2) at time $t$ and on a double Fourier collocation grid partitioning $R$,

$$
\begin{aligned}
& \left\{x_{k}=x_{\min }+\left(x_{\max }-x_{\min }\right) k / N_{x}: k=0, \ldots, N_{x}-1\right\} \\
& \left\{y_{\ell}=y_{\min }+\left(y_{\max }-y_{\min }\right) \ell / N_{y}: \ell=0, \ldots, N_{y}-1\right\} .
\end{aligned}
$$

To compute the error associated with an array $F_{k \ell}\left(t_{\mathrm{f}}\right)$ of approximate values at the final time, we first generate a reference solution

$$
F_{i j}^{\mathrm{ref}}\left(t_{\mathrm{f}}\right) \simeq \underset{5}{F}\left(t_{\mathrm{f}}, x_{i}^{\mathrm{ref}}, y_{j}^{\mathrm{ref}}\right)
$$


through a quadrature-based computation of (2). The values $F_{i j}^{\text {ref }}\left(t_{\mathrm{f}}\right)$ are defined on a uniform reference grid $\left\{\left(x_{i}^{\mathrm{ref}}, y_{j}^{\mathrm{ref}}\right): i, j=0, \ldots, N^{\mathrm{ref}}-1\right\}$, the same as (16) with $N_{x}=N^{\mathrm{ref}}=N_{y}$. More precisely, to compute each value $F_{i j}^{\text {ref }}\left(t_{\mathrm{f}}\right)$, we have used the formula (2) with $N_{\phi^{-}}$ point trapezoidal quadrature in $\phi$ and $N_{c}$-subinterval composite Gauss-Kronrod quadrature in $\tau$. The quadrature parameters $\left(N_{\phi}, N_{c}\right)$ have been chosen to ensure that the computed values $F_{i j}^{\mathrm{ref}}\left(t_{\mathrm{f}}\right)$ are of sufficient accuracy to determine the results reported below. We stress that computation of the reference solution makes no use of the rectangle $R$. Nor does it involve periodic boundary conditions (imposed in both of our methods). Figure 2 depicts the reference solution $F_{i j}^{\text {ref }}\left(t_{\mathrm{f}}\right)$ corresponding to $R=[-8.8,11.2] \times[-10.1,9.9], t_{\mathrm{f}}=5$, and $N^{\text {ref }}=256$ (although plotted on a slightly coarser grid). This solution has been generated with $\left(N_{\phi}, N_{c}\right)=(115,25)$. We will not compare the computational time of direct integration to the computational time of $F_{k \ell}\left(t_{\mathrm{f}}\right)$ by our two methods; it is significantly more expensive, even ignoring the issue that in our target application $F_{k \ell}(t)$ is needed for a range of times between 0 and $t_{\mathrm{f}}$ (i.e. the direct integration would be carried out for each intermediate time).

Since errors are always computed at time $t_{f}$, we now suppress this dependence. Comparison of $F_{k \ell}$ and $F_{i j}^{\text {ref }}$ requires interpolation. Precisely, through trigonometric interpolation, we

first extend $F_{k \ell}$ to a grid function $F_{i j}^{\text {interp }}$ defined on the reference grid $\left(x_{i}^{\text {ref }}, y_{j}^{\text {ref }}\right)$, where the assumption is that the grid (16) associated with $F_{k \ell}$ is coarser than (or perhaps the same as) the reference grid. The root-mean-square norm and error of $F_{k \ell}$ are then

$$
\operatorname{norm}(F):=\left(\frac{\sum_{i, j=0}^{N^{\mathrm{ref}}-1}\left|F_{i j}^{\mathrm{interp}}\right|^{2}}{\left(N^{\mathrm{ref}}\right)^{2}}\right)^{1 / 2}, \quad \operatorname{error}(F):=\frac{\left(\sum_{i, j=0}^{N^{\mathrm{ref}}-1}\left|F_{i j}^{\text {interp }}-F_{i j}^{\mathrm{ref}}\right|^{2}\right)^{1 / 2}}{\left(\sum_{i, j=0}^{N_{\text {ref }}-1}\left|F_{i j}^{\mathrm{interp}}\right|^{2}\right)^{1 / 2}}
$$

We likewise consider a maximum pointwise error

$$
\operatorname{error}_{\infty}(F):=\max _{0 \leq i, j \leq N^{\text {ref }}-1}\left|F_{i j}^{\text {interp }}-F_{i j}^{\mathrm{ref}}\right| .
$$

All numerical results presented here have been obtained via single-core simulations on a 2.6GHz AMD Opteron 252.

\section{First method for evaluation of (1)}

2.1. Sum-of-exponentials approximation of $J_{0}(t)$. Our first strategy for evaluation of (1) is based on (6). We now assume an approximation of the zero-order Bessel function as a sum of exponentials:

$$
J_{0}(t) \simeq A(t):=\sum_{q=1}^{d} \gamma_{q} \exp \left(\beta_{q} t\right),
$$

with the details of how such an approximation is constructed found in Appendix A. Here the locations $\beta_{q}$ and corresponding strengths $\gamma_{q}$ are complex. Furthermore, for our approximations $d=2 d_{\text {pair }}$ with the $\left(\gamma_{q}, \beta_{q}\right)$ coming in $d_{\text {pair }}$ complex-conjugate pairs, so $A(t)$ is real. We therefore change notation and denote these by $\left\{\left(m_{j}, k_{j}\right)\right\}_{j=1}^{d_{\text {pair }}}$. Here the $m_{j}=m_{j}^{R}+\mathrm{i} m_{j}^{I}$ and $k_{j}=k_{j}^{R}+\mathrm{i} k_{j}^{I}$ correspond to those complex $\left(\gamma_{q}, \beta_{q}\right)$ for which $\beta_{q}$ has positive imaginary part. Appendix A lists two specific approximations. 


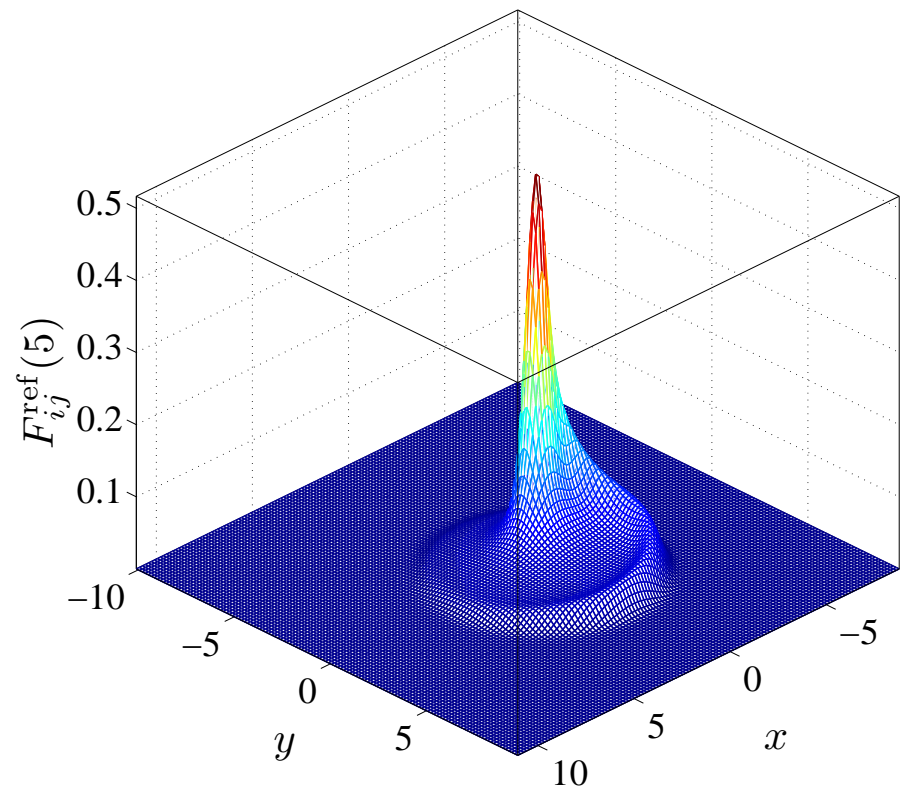

Figure 2. Reference solution at $t_{\mathrm{f}}=5$. Here the source (bunch) is approximately centered at $(-0.64091,0.71817)$.

The original sum of exponentials can now be written as

$$
\sum_{q=1}^{d} \gamma_{q} e^{\beta_{q} t}=\sum_{j=1}^{d_{\text {pair }}}\left(m_{j}^{R} C_{j}(t)-m_{j}^{I} S_{j}(t)\right),
$$

where $C_{j}(t):=2 \exp \left(k_{j}^{R} t\right) \cos \left(k_{j}^{I} t\right)$ and $S_{j}(t):=2 \exp \left(k_{j}^{R} t\right) \sin \left(k_{j}^{I} t\right)$. Informally, the stem letters here stand for cosine and sine. Now define, for example,

$$
\Psi_{j}^{C}(t, \boldsymbol{\xi} ; \widehat{f})=\int_{0}^{t} d \tau \widehat{f}(\tau, \boldsymbol{\xi}) C_{j}(\xi(t-\tau)),
$$

and likewise for $\Psi_{j}^{S}(t, \boldsymbol{\xi} ; \widehat{f})$. In terms of this notation

$$
\widehat{F}(t, \boldsymbol{\xi}) \simeq \sum_{j=1}^{d_{\text {pair }}}\left[m_{j}^{R} \Psi_{j}^{C}(t, \boldsymbol{\xi} ; \widehat{f})-m_{j}^{I} \Psi_{j}^{S}(t, \boldsymbol{\xi} ; \widehat{f})\right] .
$$

The convolutions $\Psi_{j}^{C}$ and $\Psi_{j}^{S}$ obey the following ODE system:

$$
\begin{aligned}
\frac{d}{d t} \Psi_{j}^{C}(t, \boldsymbol{\xi} ; \widehat{f}) & =k_{j}^{R} \xi \Psi_{j}^{C}(t, \boldsymbol{\xi} ; \widehat{f})-k_{j}^{I} \xi \Psi_{j}^{S}(t, \boldsymbol{\xi} ; \widehat{f})+2 \widehat{f}(t, \boldsymbol{\xi}) \\
\frac{d}{d t} \Psi_{j}^{S}(t, \boldsymbol{\xi} ; \widehat{f}) & =k_{j}^{R} \xi \Psi_{j}^{S}(t, \boldsymbol{\xi} ; \widehat{f})+k_{j}^{I} \xi \Psi_{j}^{C}(t, \boldsymbol{\xi} ; \widehat{f}) .
\end{aligned}
$$


Since the constituent convolutions $\Psi_{j}^{C}$ and $\Psi_{j}^{S}$ obey a system of ODE, we have effectively localized (6) in time. The integral over history has been removed and $\widehat{F}(t+\Delta t, \cdot)$ is approximately computed from information only at time $t$. However, (6) is an expression in the spatial frequency domain; therefore, the approach is still nonlocal in space. ${ }^{2}$

Due to the other complications in our approach, we choose to integrate (24) via the most common timestepper: classical Runge Kutta 4 (RK4). To prepare for this integration, we first approximate in space and introduce the Fourier collocation grids (16). The double discrete Fourier transform,

$$
\widehat{f}_{p q}(t)=\frac{1}{\sqrt{N_{x} N_{y}}} \sum_{k=0}^{N_{x}-1} \sum_{\ell=0}^{N_{y}-1} \exp \left(\frac{\mathrm{i} k p \pi}{N_{x}}+\frac{\mathrm{i} \ell q \pi}{N_{y}}\right) f_{k \ell}(t),
$$

with $0 \leq p \leq N_{x}-1$ and $0 \leq q \leq N_{y}-1$, then yields the corresponding discrete Fourier (modal) coefficients $\widehat{f}_{p q}$ from the nodal values $f_{k \ell}$. Likewise, we may also consider modal $\widehat{F}_{p q}$ and nodal $F_{k \ell}$ coefficients, although now the $\widehat{F}_{p q}$ are computed via our numerical method with the $F_{k \ell}$ subsequently determined by the inverse transformation to (25).

Using RK4, our approach is then to evolve the ODE (24) at the following discrete locations in the modal space:

$$
\boldsymbol{\xi}_{p q}=\left(\frac{2 \pi[p]}{x_{\max }-x_{\min }}, \frac{2 \pi[q]}{y_{\max }-y_{\min }}\right),
$$

where (and similarly for $[q]$ )

$$
2[p]= \begin{cases}p & \text { for even } p \\ p+1 & \text { for odd } p\end{cases}
$$

For this evolution one of the evolved variables is, for example, [cf. (22)]

$$
\Psi_{j, p q}^{C}(t)=\int_{0}^{t} d \tau \widehat{f}_{p q}(\tau) C_{j}\left(\left|\boldsymbol{\xi}_{p q}\right|(t-\tau)\right) .
$$

The ODE integration only requires that we have access to $\widehat{f}_{p q}(t)$, and these modal coefficients can be computed on the fly using the FFT. At any stage of the evolution, we may also compute

$$
\widehat{F}_{p q}(t)=\sum_{j=1}^{d_{\text {pair }}}\left[m_{j}^{R} \Psi_{j, p q}^{C}(t)-m_{j}^{I} \Psi_{j, p q}^{S}(t)\right],
$$

from which the nodal values $F_{k \ell}(t)$ may then be recovered with the inverse FFT.

2.2. Numerical tests. We test the method on the source function (14) for the scenario described in Section 1.2.2. The kernel approximation $A(t)$ appearing in (20) corresponds to one of the $T=10^{4}$ tables listed in Table 4 from Appendix A. As described more fully in the Appendix, each of these tables determines an $A(t)$ which approximates $J_{0}(t)$ with uniform accuracy (the $\varepsilon$ in Table 4) for non-negative times less than $10^{4}$. We stress that capital $T$ must be larger than $t_{\mathrm{f}} \xi_{\max }$ in the application. To verify that these tables are suitable for

\footnotetext{
${ }^{2}$ At slightly different approach was proposed in [4], where we considered the initial value problem for (24) at $t=t_{0}$ and used variation of parameters to obtain a solution representation in terms of a source integral over the interval $\left[t_{0}, t_{0}+\Delta t\right]$.
} 


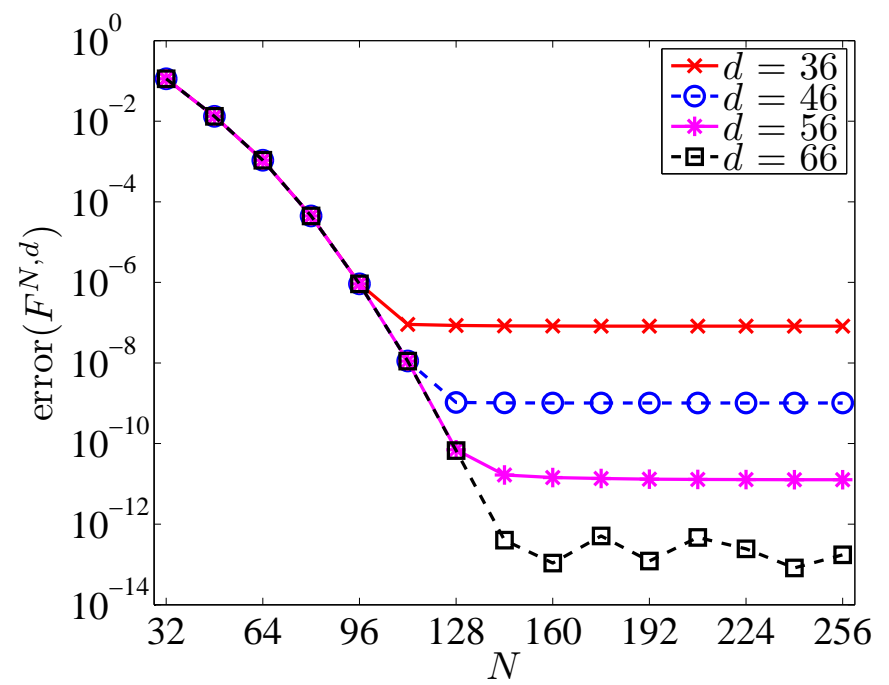

Figure 3. Relative $L_{2}$ errors computed via Method 1. As discussed in the text, for $d=36,46,56,66$ the number $n_{\text {steps }}$ of timesteps has been set to $5 N$ (crosses), $15 N$ (circles), $30 N$ (asterisks), $90 N$ (squares).

our numerical scenario, we show that $10^{4}$ bounds the discrete form of the Bessel function argument $\xi(t-\tau)$ in (6). From Eq. (26) with $N_{x}=N=N_{y} \leq 256$, the discrete wave number $\boldsymbol{\xi}_{255,255}$ corresponds to the largest possible magnitude $\left|\boldsymbol{\xi}_{255,255}\right| \simeq 56.869$. Therefore, the maximum argument $\xi(t-\tau)$ does not exceed $t_{\mathrm{f}}\left|\boldsymbol{\xi}_{255,255}\right| \simeq 5 \cdot 56.869 \ll 10^{4}$.

The computed nodal values $F_{k \ell}\left(t_{\mathrm{f}}\right)$ depend on both $N$ and the number $d$ of exponentials in the approximate kernel $A(t)$. To highlight this dependence, we now denote these numerical values by $F_{k \ell}^{N, d}\left(t_{\mathrm{f}}\right)$. The values $F_{k \ell}\left(t_{\mathrm{f}}\right)$ also depend on the chosen time step $\Delta t$ for the RK4 integration, but we will not include $\Delta t$ in the notation. Because Method 1 relies on the discrete Fourier transform, the optimal mesh resolutions $N$ are powers of 2 , but we consider the values $N \in\{32: 16: 256\}$. To compute errors, we have generated the reference solution (17) on an $N^{\text {ref }}=512$ grid.

We consider two experiments, the first examining accuracy and the second speed. Again, since all comparisons are made at the final time $t_{\mathrm{f}}=5$, we again suppress the $t_{\mathrm{f}}$ dependence. For the first experiment we compute the values $F_{k \ell}^{N, d}$ over all choices of $N$ and $d$. Here the number $n_{\text {steps }}$ of timesteps $\Delta t=t_{\mathrm{f}} / n_{\text {steps }}=5 / n_{\text {steps }}$ is determined by $N$ and $d$ as follows: for $d=36,46,56,66$ we respectively choose $n_{\text {steps }}=5 N, 15 N, 30 N, 90 N$. These choices ensure both stability and that computed values $F_{k \ell}^{N, d}$ are either dominated by spatial error or error in the approximation of $J_{0}(t)$. Figure 3 shows the corresponding relative $L_{2}$ errors as measured by (18) against the reference solution. A large number $n_{\text {steps }}$ of timesteps (small $\Delta t$ ) is necessary for the larger $N$ in order to a achieve minimal error. Indeed, our approach is spectrally accurate in space, but, owing to the chosen RK4 timestepper, only fourth-order accurate in time. With the chosen timesteps, the figure depicts the spectral convergence in space, which in each case "bottoms out" at a floor determined by $d$, a proxy for the accuracy in our sum-of-exponential approximations of $J_{0}(t)$. We believe that the choppy behavior in the $d=66$ stems from finite machine precision effects. 
Results for the second experiment appear in Table 1, which lists (both maximum pointwise and relative $L_{2}$ ) errors and the total CPU time for select parameter sets. For $N=32, \ldots, 160$, we have referred to Fig. 3 in order to choose the smallest $d$ value consistent with the requirement that the set achieves the smallest possible error. As before, the number of time steps must increase linearly with $N$ to maintain stability, and we have determined $n_{\text {steps }}$ by $N$ using the prescription described above. The values $N=176, \cdots, 256$ correspond to the regime where the error has saturated, and neither $d$ nor the number of timesteps changes. In this regime the CPU time is dominated by the cost of the double discrete Fourier transform.

\begin{tabular}{|c|c|c|c|c|c|}
\hline$N$ & $d$ & $n_{\text {steps }}$ & $\operatorname{error}_{\infty}\left(F^{N, d}\right)$ & $\operatorname{error}\left(F^{N, d}\right)$ & time $(\mathrm{sec})$ \\
\hline 32 & 36 & 160 & $7.9413 \mathrm{e}-02$ & $1.1266 \mathrm{e}-01$ & 0.5 \\
48 & 36 & 240 & $6.4046 \mathrm{e}-03$ & $1.3255 \mathrm{e}-02$ & 2.2 \\
64 & 36 & 320 & $8.8386 \mathrm{e}-04$ & $1.0717 \mathrm{e}-03$ & 5.5 \\
80 & 36 & 400 & $2.5704 \mathrm{e}-05$ & $4.4709 \mathrm{e}-05$ & 10.7 \\
96 & 36 & 480 & $4.1383 \mathrm{e}-07$ & $9.2446 \mathrm{e}-07$ & 18.5 \\
112 & 46 & 1680 & $6.7194 \mathrm{e}-09$ & $1.1222 \mathrm{e}-08$ & 128.0 \\
128 & 56 & 3840 & $3.5080 \mathrm{e}-11$ & $7.0081 \mathrm{e}-11$ & 470.2 \\
144 & 66 & 12960 & $1.8546 \mathrm{e}-13$ & $3.9941 \mathrm{e}-13$ & 2642.3 \\
160 & 66 & 14400 & $4.2966 \mathrm{e}-14$ & $1.0791 \mathrm{e}-13$ & 3613.8 \\
176 & 66 & 14400 & $6.4615 \mathrm{e}-14$ & $1.1774 \mathrm{e}-13$ & 4577.4 \\
192 & 66 & 14400 & $5.9258 \mathrm{e}-14$ & $1.1707 \mathrm{e}-13$ & 4706.4 \\
208 & 66 & 14400 & $6.4559 \mathrm{e}-14$ & $1.2084 \mathrm{e}-13$ & 5705.9 \\
224 & 66 & 14400 & $6.3866 \mathrm{e}-14$ & $1.2401 \mathrm{e}-13$ & 8041.5 \\
240 & 66 & 14400 & $5.9119 \mathrm{e}-14$ & $1.2150 \mathrm{e}-13$ & 9802.7 \\
256 & 66 & 14400 & $4.1023 \mathrm{e}-14$ & $1.0787 \mathrm{e}-13$ & 11844.0 \\
\hline
\end{tabular}

TABle 1. MAXimum errors and CPU times.

2.3. Error estimate. Here we provide a theoretical argument suggesting that (30)

$$
\left[\left\|F^{N, d(\varepsilon)}(t)\right\| \cdot \operatorname{error}\left(F^{N, d(\varepsilon)}(t)\right)\right]^{2} \lesssim \overbrace{N^{-p_{0}} C_{0}(t)}^{\text {Fourier truncation }}+\overbrace{N^{-p_{1}} C_{1}(t)}^{\text {aliasing }}+\overbrace{\varepsilon C_{2}(t, N)+\varepsilon^{2} C_{3}(t)}^{\text {aliasing and kernel approximation }},
$$

where $p_{0}$ and $p_{1}$ are positive integers determined respectively by the regularity of $F(t, \cdot)$ and $f(t, \cdot)$. As argued below, we expect that the factor $C_{2}(t, N)$ decays with $N$. The notation $d(\varepsilon)$ on the left-hand side of (30) stresses that the number $d$ of exponential terms in the approximation (20) depends on the chosen tolerance $\varepsilon$. Moreover, the 2-norm $\left\|F^{N, d}(t)\right\|$ appears since error $\left(F^{N, d}(t)\right)$ is defined as a relative error, the continuum analog of the discrete error in (18). The scaling behavior (30) comports with the results in Fig. 3, where the error falls off with $N$ until a floor is reached (the last term in the formula). The rapid decay with $N$ indicates that $p_{0}$ and $p_{1}$ are arbitrarily large due to the smoothness of our test problem. The simple scaling (30) ignores the temporal error associated with the Runge-Kutta evolution of (the constituent pieces of) the convolution. For our simulations, the timestep $\Delta t$ is always chosen small enough to ensure that this temporal error is subdominant. In principal (30) 
allows for optimal prediction of the required number $N$ of Fourier modes in each direction; the idea being to choose $N$ such that the first two terms are the same size as the last. However, such prediction would ideally rely on knowledge of $p_{0}, p_{1}$, and the $C$ constants.

For simplicity, assume now that the domain is $R=[0,2 \pi]^{2}$, and that $f(t, \cdot)$ and $F(t, \cdot)$ are smooth and compactly supported on $R$ for $0 \leq t \leq t_{F}$. The spatial Fourier transform and Fourier coefficients of $F(t, \cdot)$ are then given by

$$
\widehat{F}(t, \boldsymbol{\xi})=\frac{1}{2 \pi} \int_{[0,2 \pi]^{2}} d \mathbf{x} e^{-\mathrm{i} \mathbf{x} \cdot \boldsymbol{\xi}} F(t, \mathbf{x}), \quad \bar{F}_{n m}(t) \equiv \bar{F}_{\boldsymbol{n}}(t)=\frac{1}{2 \pi} \widehat{F}(t, \boldsymbol{n}),
$$

where $\boldsymbol{n}=(n, m)$. Whereas inverse Fourier transformation of $\widehat{F}(t, \cdot)$ yields the original compactly supported $F(t, \cdot)$, summation of the series on the Fourier basis yields the doubly periodic extension of $F(t, \cdot)$ restricted to $R$. The overbar on $\bar{F}_{n m}$ distinguishes these coefficients from the $F_{k \ell}$ introduced after (25). All of these comments and formulas of course also pertain to $f(t, \cdot)$.

Consider the trigonometric polynomial stemming from the truncated Fourier series (the $\mathcal{P}$ notation is from [14]),

$$
\mathcal{P}_{N} F(t, \mathbf{x})=\sum_{|n|,|m| \leq N / 2} \bar{F}_{n m}(t) e^{\mathrm{i}(n x+m y)},
$$

with the same even truncation $N$ chosen in each direction for simplicity. Numerically, what we actually compute is

$$
F^{\mathrm{C}}(t, \mathbf{x})=\sum_{|n|,|m| \leq N / 2} \bar{F}_{n m}^{\mathrm{C}}(t) e^{\mathrm{i}(n x+m y)}
$$

where the $\mathrm{C}$ stands for computed. Note that the $\bar{F}_{n m}^{\mathrm{C}}$ (defined below) are not the $\widehat{F}_{p q}$ introduced after (25). In particular, the indices on $\widehat{F}_{p q}$ run from 0 to $N-1$, whereas the indices on $\bar{F}_{n m}^{\mathrm{C}}$ run from $-N / 2$ to $N / 2$. Moreover, there are also differences due to scalings by factors of $N$. The relationship is the same as given below in (36).

Our justification of (30) starts with the formula

$$
\left\|F(t, \cdot)-F^{\mathrm{C}}(t, \cdot)\right\|_{L_{2}(R)}^{2}=\overbrace{\left\|F(t, \cdot)-\mathcal{P}_{N} F(t, \cdot)\right\|_{L_{2(R)}}^{2}}^{(\text {term } 1)}+\overbrace{\left\|\mathcal{P}_{N} F(t, \cdot)-F^{\mathrm{C}}(t, \cdot)\right\|_{L_{2(R)}}^{2}}^{(\text {term 2)}},
$$

which holds because $F(t, \cdot)-\mathcal{P}_{N} F(t, \cdot)$ is orthogonal to $\mathcal{P}_{N} F(t, \cdot)-F^{\mathrm{C}}(t, \cdot)$ in the $L_{2}(R)$-inner product. The left-hand sides of (30) and the last formula correspond because error $\left(F^{N, d}\right)$ is a relative 2 -norm error. Now, (term 1 ) is amenable to classical estimates, yielding the first term on the right-hand side of (30). Our remaining goal is then to establish that the dominant sources of error in (term 2) can be expressed as the final three terms in (30).

The numerically computed coefficients are

$$
\bar{F}_{n m}^{\mathrm{C}}(t)=\int_{0}^{t} d \tau \bar{f}_{n m}^{\mathrm{D}}(\tau) A(|\boldsymbol{n}|(t-\tau)),
$$

where $A(t)$ is the sum-of-exponentials approximation to $J_{0}(t)$ and the $\bar{f}_{n m}^{\mathrm{D}}$ are the discrete Fourier coefficients (computed by the Trapezoid Rule) of the source $f(t, \cdot)$. As before, these 
are not the coefficients $\widehat{f}_{p q}$ introduced above, rather [14]

$$
\widehat{f}_{p q}=N \gamma_{n(p)} \gamma_{m(q)} \bar{f}_{n(p), m(q)}^{\mathrm{D}}, \quad \gamma_{k}= \begin{cases}1 & \text { for }|k|<N / 2 \\ 2 & \text { for }|k|=N / 2\end{cases}
$$

Here, for example, $n(p)=p$ for $p=0, \ldots,-1+N / 2$ and $n(p)=p-N$ for $p=N / 2, \ldots, N-1$. This distinction will be familiar to those experienced in using the discrete Fourier transform. Moreover, here we use $\bar{f}_{n m}^{\mathrm{D}}$ (not $\bar{f}_{n m}^{\mathrm{C}}$ ), because the $\bar{f}_{n m}^{\mathrm{D}}$ are indeed the discrete Fourier coefficients stemming from the exact $f(t, \cdot)$ in our model problem. Parseval's formula yields

$$
(\operatorname{term} 2)=4 \pi^{2} \sum_{|n|,|m|<N / 2}\left|\bar{F}_{n m}^{\mathrm{C}}-\bar{F}_{n m}\right|^{2},
$$

where we may express the terms in the sum as

$$
\bar{F}_{n m}^{\mathrm{C}}(t)-\bar{F}_{n m}(t)=\int_{0}^{t} d \tau\left[\bar{f}_{n m}^{\mathrm{D}}(\tau)-\bar{f}_{n m}(\tau)\right] J_{0}(|\boldsymbol{n}|(t-\tau))+\int_{0}^{t} d \tau \bar{f}_{n m}^{\mathrm{D}}(\tau) \Delta J_{0}(|\boldsymbol{n}|(t-\tau)),
$$

where $\Delta J_{0}(t) \equiv A(t)-J_{0}(t)$. Since $J_{0}(t) \leq 1$, we then have

$$
\left|\bar{F}_{n m}^{\mathrm{C}}(t)-\bar{F}_{n m}(t)\right| \leq \int_{0}^{t} d \tau\left|\bar{f}_{n m}^{\mathrm{D}}(\tau)-\bar{f}_{n m}(\tau)\right|+\max _{\tau \in[0, T]}\left|\Delta J_{0}(\tau)\right| \cdot \int_{0}^{t} d \tau\left|\bar{f}_{n m}^{\mathrm{D}}(\tau)\right|,
$$

where $T=\sqrt{2} N t$ in terms of the maximum size $\sqrt{2} N$ of $|\boldsymbol{n}|$. Finally, we square the last equation, and then invoke the Cauchy-Schwarz inequality on the right-hand side, finding

$$
\begin{aligned}
\left|\bar{F}_{n m}^{\mathrm{C}}(t)-\bar{F}_{n m}(t)\right|^{2} & \leq t \int_{0}^{t} d \tau\left|\bar{f}_{n m}^{\mathrm{D}}(\tau)-\bar{f}_{n m}(\tau)\right|^{2}+t\left[\max _{\tau \in[0, T]}\left|\Delta J_{0}(\tau)\right|\right]^{2} \cdot \int_{0}^{t} d \tau\left|\bar{f}_{n m}^{\mathrm{D}}(\tau)\right|^{2} \\
& +2 \max _{\tau \in[0, T]}\left|\Delta J_{0}(\tau)\right| \cdot \int_{0}^{t} d \tau\left|\bar{f}_{n m}^{\mathrm{D}}(\tau)\right| \cdot \int_{0}^{t} d \tau\left|\bar{f}_{n m}^{\mathrm{D}}(\tau)-\bar{f}_{n m}(\tau)\right| .
\end{aligned}
$$

Summing over $|n|,|m| \leq N / 2$ and multiplying by $4 \pi^{2}$, we then reach

$$
\begin{aligned}
(\operatorname{term} 2) & \leq t\left\|\mathcal{I}_{N} f(t, \cdot)-\mathcal{P}_{N} f(t, \cdot)\right\|_{L_{2}(R)}^{2}+t\left[\max _{\tau \in[0, T]}\left|\Delta J_{0}(\tau)\right|\right]^{2} \cdot\left\|\mathcal{I}_{N} f(t, \cdot)\right\|_{L_{2}(R)}^{2} \\
& +8 \pi^{2} \max _{\tau \in[0, T]}\left|\Delta J_{0}(\tau)\right| \sum_{|n|,|m| \leq N / 2} \int_{0}^{t} d \tau\left|f_{n m}^{\mathrm{D}}(\tau)\right| \cdot \int_{0}^{t} d \tau\left|\bar{f}_{n m}^{\mathrm{D}}(\tau)-\bar{f}_{n m}(\tau)\right| .
\end{aligned}
$$

Here we have introduced the notation (again, see [14])

$$
\mathcal{I}_{N} f(t, \mathbf{x})=\sum_{|n|,|m| \leq N / 2} f_{n m}^{\mathrm{D}} e^{\mathrm{i}(n x+m y)} .
$$

We identify the first term in (41) as aliasing error; it corresponds to the second term on the right-hand side of (30). Appendix A demonstrates empirically that our rational approximations satisfy $\max _{\tau \in[0, T]} \mid \max \left(1, \sqrt{(1 / 2) \pi \tau} \Delta J_{0}(\tau) \mid<\varepsilon\right.$. With this observation, we infer the final two terms in (30). Since in (41) the second integral in the last term also involves aliasing error, we conjecture that $C_{2}(t, N)$ decays with $N$. 


\section{Second method for evaluation of (1)}

Method 2 involves evolution of the time-dependent $3+1$ wave equation $\square U=0$ above the sheet $z=0$. Here the solution $U(t, \mathbf{x}, z)$ is the function in (13). According to (11), the integral (1) we wish to compute is the restriction $U(t, \mathbf{x}, 0)$ to $z=0$ of the unique solution to the IBVP (12). Our numerical method for computing $U(t, \mathbf{x}, 0)$ requires appropriate boundary conditions both on the $z=0$ sheet (simply the condition $\partial_{z} U=-f$ specified in the open problem) and on the artificial planar boundary $z=\delta$. One option would be to choose $\delta$ large enough to ensure that for $t \leq t_{\mathrm{f}}$ the $z=\delta$ boundary is causally disconnected from the sheet. In this case the values $U(t, \mathbf{x}, 0)$ would be insensitive to the distant boundary conditions, provided only that they were stable. However, this option would prove prohibitively costly. Therefore, we choose $\delta$ not large and adopt radiation boundary conditions (described below) developed by Hagstrom, Warburton, and Givoli [5] and Hagstrom and Warburton [6]. We take the interior of the "computational domain" to be the region

$$
\mathcal{D}=\left\{\mathbf{X}=(\mathbf{x}, z): \mathbf{x} \in \mathbb{R}^{2}, 0<z<\delta\right\} .
$$

In practice, we must also truncate in the transverse directions; however, as discussed further below, in the numerical implementation we have simply enforced periodicity in $x$ and $y$. Nevertheless, for the time being we consider the idealization of "open" transverse directions. In order to describe the exact radiation boundary conditions at $z=\delta$ (as well as their numerical approximation), we proceed by reformulating the problem (12) in terms of a firstorder symmetric hyperbolic (FOSH) system based on characteristic variables.

\subsection{First-order symmetric hyperbolic (FOSH) system. Define the system vector}

$$
\mathbf{W}=\left(\begin{array}{c}
\mathbf{w} \\
U
\end{array}\right), \quad \mathbf{w}=\left(W^{+}, W^{-}, \Phi_{1}, \Phi_{2}\right)^{t},
$$

where

$$
W^{+}=\frac{1}{\sqrt{2}}\left(-\partial_{t} U+\partial_{z} U\right), \quad W^{-}=\frac{1}{\sqrt{2}}\left(-\partial_{t} U-\partial_{z} U\right), \quad \Phi_{1}=\partial_{x} U, \quad \Phi_{2}=\partial_{y} U .
$$

We sometimes write $\boldsymbol{\Phi}$ for $\left(\Phi_{1}, \Phi_{2}\right)^{t}$, and also $\Phi_{a}=\partial_{a} U$ for $a=1,2$. The equations of motion for these fields are

$$
\begin{aligned}
\partial_{t} W^{+} & =-\partial_{z} W^{+}-\frac{1}{\sqrt{2}}\left(\partial_{x} \Phi_{1}+\partial_{y} \Phi_{2}\right) \\
\partial_{t} W^{-} & =+\partial_{z} W^{-}-\frac{1}{\sqrt{2}}\left(\partial_{x} \Phi_{1}+\partial_{y} \Phi_{2}\right) \\
\partial_{t} \boldsymbol{\Phi} & =-\frac{1}{\sqrt{2}} \nabla\left(W^{+}+W^{-}\right) \\
\partial_{t} U & =-\frac{1}{\sqrt{2}}\left(W^{+}+W^{-}\right) .
\end{aligned}
$$

Here we use $\nabla=\left(\partial_{x}, \partial_{y}\right)^{t}$ as the gradient in the tangential variables (the three-dimensional gradient does not appear in this article). Our motivation for the definitions (45) is as follows. Define the characteristic (null) vector fields $e_{ \pm}=2^{-1 / 2}\left(\partial_{t} \pm \partial_{z}\right)$. We then use $W_{ \pm}=e_{ \pm}[U]$ for the associated characteristic derivatives, and finally set $W^{ \pm}=-W_{\mp}$. The last formula is consistent with raising the \pm index by a Minkowski metric of signature 2 . With these 
conventions the variable $W^{+}$propagates along $e_{+}$, and the variable $W^{-}$along $e_{-}$. Ignoring the time direction and with the $\partial / \partial z$ direction viewed as straight up the page, $W^{+}$propagates in the $\uparrow$ direction, and the variable $W^{-}$in the $\downarrow$ direction. Our $W^{ \pm}$agree with the literature referenced at [12] and Hagstrom-Warburton [6], although $W^{ \pm, \text {here }}=w_{ \pm, \mathrm{HW}}$.

In matrix form, the system (46) is written as

$$
L \mathbf{W}=0, \quad L:=\frac{\partial}{\partial t}+A \frac{\partial}{\partial z}+B_{1} \frac{\partial}{\partial x}+B_{2} \frac{\partial}{\partial y}+C,
$$

where the matrices in this symmetric hyperbolic system are as follows:

$$
\begin{aligned}
& A=\left(\begin{array}{ccccc}
1 & 0 & 0 & 0 & 0 \\
0 & -1 & 0 & 0 & 0 \\
0 & 0 & 0 & 0 & 0 \\
0 & 0 & 0 & 0 & 0 \\
0 & 0 & 0 & 0 & 0
\end{array}\right), \quad B_{1}=\left(\begin{array}{ccccc}
0 & 0 & 2^{-1 / 2} & 0 & 0 \\
0 & 0 & 2^{-1 / 2} & 0 & 0 \\
2^{-1 / 2} & 2^{-1 / 2} & 0 & 0 & 0 \\
0 & 0 & 0 & 0 & 0 \\
0 & 0 & 0 & 0 & 0
\end{array}\right) \\
& C=\left(\begin{array}{ccccc}
0 & 0 & 0 & 0 & 0 \\
0 & 0 & 0 & 0 & 0 \\
0 & 0 & 0 & 0 & 0 \\
0 & 0 & 0 & 0 & 0 \\
2^{-1 / 2} & 2^{-1 / 2} & 0 & 0 & 0
\end{array}\right), \quad B_{2}=\left(\begin{array}{ccccc}
0 & 0 & 0 & 2^{-1 / 2} & 0 \\
0 & 0 & 0 & 2^{-1 / 2} & 0 \\
0 & 0 & 0 & 0 & 0 \\
2^{-1 / 2} & 2^{-1 / 2} & 0 & 0 & 0 \\
0 & 0 & 0 & 0 & 0
\end{array}\right) .
\end{aligned}
$$

With (47) reformulate (12) as

$$
\begin{aligned}
& L \mathbf{W}=0, t>0, \mathbf{X} \in \mathbb{R}_{+}^{3} \\
& \mathbf{W}=0, \quad t=0, \mathbf{X} \in \mathbb{R}_{+}^{3} \\
& W^{+}=W^{-}-\sqrt{2} f, \quad t>0, \mathbf{X} \in \partial \mathbb{R}_{+}^{3} .
\end{aligned}
$$

The condition (49b) of trivial initial data ensures that on the $t=0$ initial surface for $z>0$ each of the following constraints vanishes (again where $a=1,2$ ):

$$
\begin{aligned}
\mathcal{C}_{a} & :=\Phi_{a}-\partial_{a} U \\
\mathcal{C}_{3} & :=2^{-1 / 2}\left(W^{+}-W^{-}\right)-\partial_{z} U \\
\mathcal{C}_{a 3} & :=2^{-3 / 2} \partial_{a}\left(W^{+}-W^{-}\right)-\frac{1}{2} \partial_{z} \Phi_{a} \\
\mathcal{C}_{12} & :=\frac{1}{2}\left(\partial_{x} \Phi_{2}-\partial_{y} \Phi_{1}\right) .
\end{aligned}
$$

These expressions stem from the definitions (45). The constraints $\mathcal{C}_{i j}=0$ follow from $\mathcal{C}_{i}=0$ (here $i, j$ run over $1,2,3$ ). Moreover, the equations of motion (46) imply that $\partial_{t} \mathcal{C}_{j}=0=\partial_{t} \mathcal{C}_{i j}$; whence these constraints vanish for all $t>0$. We then have the following results relating problems (12) and (49).

Claim 3.1. If $U$ is a solution to (12), then $\mathbf{W}$ as defined in (45) is a solution to (49).

Proof. The justification amounts to straightforward substitutions.

Claim 3.2. If $\mathbf{W}$ is a solution to (49), then its last component $U$ solves (12). Moreover, the definitions (45) hold for all $t \geq 0$. 
Proof. Since $(46 \mathrm{~d})$ and the constraints $\mathcal{C}_{3}=0=\mathcal{C}_{a}$ hold for all time, we conclude that Eqs. (45) do as well. To show that $\square U=0$ in the half space, we time differentiate (46d), and then in the resulting equation eliminate the terms $\partial_{t} W^{ \pm}$using $(46 a, b)$, thereby finding

$$
\partial_{t}^{2} U=\frac{1}{\sqrt{2}} \partial_{z}\left(W^{+}-W^{-}\right)+\nabla \cdot \Phi .
$$

The result is then obtained upon further eliminations with the constraints.

Remark 3.3. Since the definitions (45) hold for all time, $\square \mathbf{W}=0$.

3.2. Radiation boundary conditions. As mentioned, it is impractical to solve the open problem, and we therefore introduce the artificial boundary $z=\delta$. Again viewing the $z$-axis as the vertical direction, $W^{+}$propagates in the $\uparrow$ direction with speed 1 , and $W^{-}$in the $\downarrow$ direction with speed 1 . The remaining variables $U, \Phi_{1}, \Phi_{2}$ propagate with speed 0 relative to the boundaries at $z=0, \delta$. Therefore, to pose the problem on $\mathcal{D}$ we must specify $W^{+}$at $z=0$ and $W^{-}$at $z=\delta$. We again adopt (49c) as the $z=0$ boundary condition. FourierLaplace analysis yields an identity (which for our problem is valid for all $z>0$ ) that will serve as the boundary condition at $z=\delta$. Indeed, Fourier-Laplace transform of the system (46) followed by recombination of the resulting equations yields

$$
\begin{aligned}
s \stackrel{\vee}{W^{ \pm}} & =\mp \frac{d}{d z} \stackrel{\vee}{W}^{ \pm}-\frac{1}{2 s} \xi^{2}\left(\breve{V}^{+}+\check{V}^{-}\right) \\
s \stackrel{\vee}{\mathbf{\Phi}} & =-\frac{1}{\sqrt{2}} \mathrm{i} \boldsymbol{\xi}\left(\stackrel{\vee}{W}^{+}+\stackrel{\vee}{W}^{-}\right) \\
s \stackrel{\vee}{U} & =-\frac{1}{\sqrt{2}}\left(\stackrel{\vee}{W}^{+}+\stackrel{\vee}{W}^{-}\right) .
\end{aligned}
$$

With $\gamma=\left(s^{2}+\xi^{2}\right)^{1 / 2}$, the general solution of (52a) is (suppressing the arguments on $\stackrel{\vee}{W}^{ \pm}$)

$$
\left(\begin{array}{c}
\breve{W}^{+} \\
\breve{W}^{-}
\end{array}\right)=\alpha(s, \boldsymbol{\xi}) e^{-\gamma z}\left(\begin{array}{c}
s+\gamma \\
s-\gamma
\end{array}\right)+\beta(s, \boldsymbol{\xi}) e^{\gamma z}\left(\begin{array}{c}
s-\gamma \\
s+\gamma
\end{array}\right) .
$$

For bounded solutions we must have $\beta(s, \boldsymbol{\xi})=0$, and the boundary condition at $z=0$ gives

$$
\alpha(s, \boldsymbol{\xi})=-\frac{1}{\sqrt{2}} \frac{\stackrel{\vee}{f}(s, \boldsymbol{\xi})}{\sqrt{s^{2}+\xi^{2}}} .
$$

Thus the unique solution of (49) in the Fourier-Laplace domain is (53) with $\beta(s, \boldsymbol{\xi})=0$ and this $\alpha(s, \boldsymbol{\xi})$, along with $(52 \mathrm{~b}, \mathrm{c})$. We work in the right-half plane $s \in T^{-1}+\mathrm{i} \mathbb{R}$, so that $s+\gamma \neq 0$ and division by this quantity makes sense (the meaning of $T$ is addressed below). Independent of the boundary condition at $z=0$, bounded solutions to (53) obey

$$
\stackrel{\vee}{W}^{-}=\stackrel{\vee}{P}(s, \boldsymbol{\xi}) \stackrel{\vee}{W}^{+}:=\frac{s-\gamma}{s+\gamma} \breve{V}^{+}, \quad \stackrel{v}{P}(s, \boldsymbol{\xi})=K\left(\xi^{-1} s\right), \quad K(\zeta)=\frac{\zeta-\sqrt{\zeta^{2}+1}}{\zeta+\sqrt{\zeta^{2}+1}} .
$$

which is our exact radiation boundary condition in the Fourier-Laplace domain. The approximate boundary conditions considered in the next subsection are tailored to approximate $P(s, \boldsymbol{\xi})$. We stress that $(55)$ holds for all $z>0$; nevertheless, we now replace $z$ by $\delta$ (an arbitrary positive number) to emphasize use of this formula as a boundary condition. 
Equation (55) is algebraically equivalent to

$$
\stackrel{\vee}{W}^{-}(s, \boldsymbol{\xi}, \delta)=-\stackrel{\vee}{W}^{+}(s, \boldsymbol{\xi}, \delta)+\stackrel{\vee}{P}_{1}(s, \boldsymbol{\xi}) s \stackrel{\vee}{W}^{+}(s, \boldsymbol{\xi}, \delta), \quad \stackrel{\vee}{P}_{1}(s, \boldsymbol{\xi})=\frac{2}{\sqrt{s^{2}+\xi^{2}}+s},
$$

where the subscript 1 on $\stackrel{\vee}{P}_{1}$ merely serves to distinguish this symbol from $\stackrel{\vee}{P}$ in (55). Appendix $\mathrm{C}$ shows that the spacetime form of (56) is the "classical" (singularity free) relationship

$$
W^{-}(t, \mathbf{x}, \delta)=-W^{+}(t, \mathbf{x}, \delta)+\frac{1}{\pi} \int_{0}^{t} d t^{\prime} \int_{0}^{1} d \varrho \varrho \int_{0}^{2 \pi} d \phi D_{0} W^{+}\left(t^{\prime}, \mathbf{x}+\left(t-t^{\prime}\right) \varrho \nu(\phi), \delta\right),
$$

where $D_{0}$ indicates differentiation in the time slot. More formally, Eq. (57) is

$$
W^{-}(t, \mathbf{x}, \delta)=-W^{+}(t, \mathbf{x}, \delta)+\int_{0}^{t} d t^{\prime} \int_{\left|\mathbf{x}-\mathbf{x}^{\prime}\right| \leq\left(t-t^{\prime}\right)} d \mathbf{x}^{\prime} \frac{D_{0} W^{+}\left(t^{\prime}, \mathbf{x}^{\prime}, \delta\right)}{\pi\left(t-t^{\prime}\right)^{2}}
$$

and this expression manifestly involves the normalized average of $D_{0} W^{+}$over the disk which arises as the intersection of the boundary $z=\delta$ and the interior of the backward light cone with apex $(t, \mathbf{x}, \delta)$. Reference [13] considers other geometrical alternatives to (58). Moreover, while here we have considered the tangential-Fourier/Laplace and spacetime forms of the boundary condition, via the Schwartz theory of distributions Ref. [13] also analyzes the tangential-Fourier/time-Fourier form (that is, $3 \mathrm{~d}$ Fourier transformation of (57) in $\mathbf{x}$ and $t$ ).

With the relation (57), we have the following.

Claim 3.4. Consider the problem (49) but now with $\mathbf{X} \in \mathcal{D}$ in (49a,b) and $\left.\mathbf{X} \in \partial \mathcal{D}\right|_{z=0}$ in (49c). Moreover, adopt (57) for $t>0,\left.\mathbf{X} \in \partial \mathcal{D}\right|_{z=\delta}$. Then for $t \geq 0$ the unique solution of the resulting problem is the restriction of the unique solution of $(49)$ from $\mathbb{R}_{+}^{3}$ to $\mathcal{D}$.

Proof. The claim is established by the analysis above which shows that

$$
\left(\begin{array}{c}
\breve{v}^{ \pm}(s, \boldsymbol{\xi}, z) \\
\stackrel{\vee}{\mathbf{\Phi}}(s, \boldsymbol{\xi}, z) \\
\stackrel{v}{U}(s, \boldsymbol{\xi}, z)
\end{array}\right)=\left(\begin{array}{c}
2^{-1 / 2}(-s \mp \gamma) \\
\mathrm{i} \boldsymbol{\xi} \\
1
\end{array}\right) \gamma^{-1} \stackrel{\vee}{f}(s, \boldsymbol{\xi}) e^{-\gamma z}
$$

is the unique solution to both problems in the Fourier-Laplace domain.

Note that the last component in (59) is the result expressed in Eq. (10) with $z>0$.

3.3. Hagstrom-Warburton-Givoli boundary condition. Following Hagstrom, Warburton, and Givoli [5, 6], we approximate the boundary condition (55) via a local, time-domain, and physical-space procedure which relates $W^{-}(t, \mathbf{x}, \delta)$ to $W^{+}(t, \mathbf{x}, \delta)$. The procedure relies on auxiliary boundary variables and their governing system. Ultimately, this boundary system is coupled to the system for the interior (or bulk) variables. However, we first introduce and discuss the properties of the boundary system in its own right.

Introduce $P+2$ auxiliary vectors $(4 P+8$ scalar variables $):\left\{\mathbf{w}_{j}=\left(W_{j}^{+}, W_{j}^{-}, \Phi_{1, j}, \Phi_{2, j}\right)^{t}\right\}_{j=0}^{P+1}$. We think of these variables as "living" on the boundary $z=\delta$, but each vector has the argument structure $\mathbf{w}_{j}=\mathbf{w}_{j}(t, \mathbf{x})$. Moreover, introduce $2 P+2$ parameters $\left\{\left(\psi_{j}, \bar{\psi}_{j}\right)\right\}_{j=0}^{P}$, whose origins will be discussed below. As shorthands, we also define $c_{j}=\cos \psi_{j}$ and $\sigma_{j}=T^{-1} \sin \psi_{j} \tan \psi_{j}$, and similarly for $\bar{c}_{j}$ and $\bar{\sigma}_{j}$. Given $T$, for each $j$ the "cosine parameters" $\left(c_{j}, \bar{c}_{j}\right)$ determine $\left(\sigma_{j}, \bar{\sigma}_{j}\right)$. $T$ will characterize the time to which our procedure 
accurately approximates the exact boundary condition (57). In terms of these quantities the auxiliary variables satisfy the following underdetermined system of $4 P+6$ equations:

$$
\begin{aligned}
& {\left[\left(1+\bar{c}_{j}\right) \partial_{t}+\bar{\sigma}_{j}\right] W_{j+1}^{+}=\left[\left(-1+c_{j}\right) \partial_{t}+\sigma_{j}\right] W_{j}^{+}-\frac{1}{\sqrt{2}} \nabla \cdot\left(\boldsymbol{\Phi}_{j}+\boldsymbol{\Phi}_{j+1}\right)} \\
& {\left[\left(1+c_{j}\right) \partial_{t}+\sigma_{j}\right] W_{j}^{-}=\left[\left(-1+\bar{c}_{j}\right) \partial_{t}+\bar{\sigma}_{j}\right] W_{j+1}^{-}-\frac{1}{\sqrt{2}} \nabla \cdot\left(\boldsymbol{\Phi}_{j}+\boldsymbol{\Phi}_{j+1}\right)} \\
& \partial_{t} \boldsymbol{\Phi}_{j}=-\frac{1}{\sqrt{2}} \nabla\left(W_{j}^{+}+W_{j}^{-}\right),
\end{aligned}
$$

where for $(60 \mathrm{a}, \mathrm{b}) j$ runs over $0, \ldots, P$, and for $(60 \mathrm{c}) j$ runs from $0, \ldots, P+1$. Notice that equations (60c) are identical to the tangential sector (46c) of the FOSH system. The following claim relates $W_{0}^{-}$to $W_{0}^{+}$, and it is a key observation underlying the use of the auxiliary system to fix a boundary condition at $z=\delta$.

Claim 3.5. Suppose that the variables $\left\{\mathbf{w}_{j}\right\}_{j=0}^{P+1}$ vanish at $t=0$ and obey the system (60). Furthermore, assume (i) the "closure condition" $W_{P+1}^{-}(t, \mathbf{x})=0$ and (ii) the prescription $W_{0}^{+}(t, \mathbf{x})=g(t, \mathbf{x})$, both conditions for all $t>0, \mathbf{x} \in \mathbb{R}^{2}$. Then $W_{0}^{-}(t, \mathbf{x})$ is uniquely determined as a function of its arguments.

Notice that (60) and the two conditions in Claim 3.5 together are $4 P+8$ equations for the $4 P+8$ auxiliary scalar variables. Proof of the claim relies on the Fourier-Laplace transform to convert the problem into a linear system of equations, and is established during the proof of Theorem 3.7, given in [6]. This theorem explicitly expresses $\stackrel{v}{W}_{0}^{-}(s, \boldsymbol{\xi})$ in terms of $\stackrel{\vee}{W}+(s, \boldsymbol{\xi})=\stackrel{\vee}{g}(s, \boldsymbol{\xi})$.

Remark 3.6. Since, in particular, we assume $\left.W_{0}^{+}\right|_{t=0}=0$, prescription of $W_{0}^{+}$is equivalent to prescription of $\partial_{t} W_{0}^{+}$.

Theorem 3.7. (Hagstrom and Warburton). Evolving the system (60) with (i) $W_{0}^{+}$viewed as a prescribed source and (ii) the closure condition $W_{P+1}^{-}=0$ corresponds to the following relationship in the Fourier-Laplace domain (A for "approximate"):

$$
\stackrel{\vee}{W}_{0}^{-}=\stackrel{\vee}{P}^{A}(s, \boldsymbol{\xi}) \stackrel{\vee}{W}_{0}^{+}, \quad \stackrel{\vee}{P}^{A}(s, \boldsymbol{\xi})=K^{A}\left(s, \gamma^{2}, T\right)
$$

where, as before, $\gamma=\sqrt{s^{2}+\xi^{2}}$ and

$$
\begin{aligned}
K^{A}\left(s, \gamma^{2}, T\right) & =-\xi^{2}\left[\frac{Q(-\gamma)-Q(\gamma)}{(s+\gamma)^{2} Q(-\gamma)-(s-\gamma)^{2} Q(\gamma)}\right] \\
Q(\zeta) & =\prod_{j=0}^{P}\left(\zeta-s c_{j}-\sigma_{j}\right)\left(\zeta-s \bar{c}_{j}-\bar{\sigma}_{j}\right) .
\end{aligned}
$$

Note that the right hand side of (62a) is even in $\gamma$; therefore, $K^{A}\left(s, \gamma^{2}, T\right)$ indeed depends on this variable through $\gamma^{2}$. Our proof of this theorem directly follows the one given by Hagstrom and Warburton [6]. However, since our artificial boundary is at $z=\delta$, whereas Hagstrom and Warburton's is at $x=0$, our expressions for the reflection coefficient (a focus 
of the proof),

$$
R(s, \boldsymbol{\xi})=\left(\frac{\gamma-s}{\gamma+s}\right) \frac{Q(\gamma)}{Q(-\gamma)}, \quad \frac{\stackrel{v}{W}_{0}^{-}}{V_{0}^{+}}=\frac{\left(\frac{s-\gamma}{s+\gamma}\right)+R(s, \boldsymbol{\xi})}{1+R(s, \boldsymbol{\xi})\left(\frac{s-\gamma}{s+\gamma}\right)},
$$

differs slightly from the one in [6].

For fixed $\eta:=\delta / T$, Hagstrom and Warburton [6] describe the selection of "optimal cosines" $\left\{c_{j}, \bar{c}_{j}\right\}_{j=0}^{P}$ to minimize the magnitude $|R(s, \boldsymbol{\xi})|$ of the reflection coefficient associated with the boundary $z=\delta$; in this viewpoint we identify $W_{0}^{ \pm}(t, \mathbf{x})$ with the restrictions $W^{ \pm}(t, \mathbf{x}, \delta)$ to the boundary $z=\delta$, in which case (61) determines a boundary condition. We return to this issue in Section 3.4. The selection of optimal cosines ensures that $K^{A}\left(s, \gamma^{2}, T\right)$ in (61) approximates $K\left(\xi^{-1} s\right)$ in (55) along the inversion contour $s \in T^{-1}+\mathrm{i} \mathbb{R}$, as verified empirically in the next paragraph. Optimal cosine tables are available at the following URL.

faculty.smu.edu/thagstrom/Optimal_cosines.txt

We use the following table from the above URL.

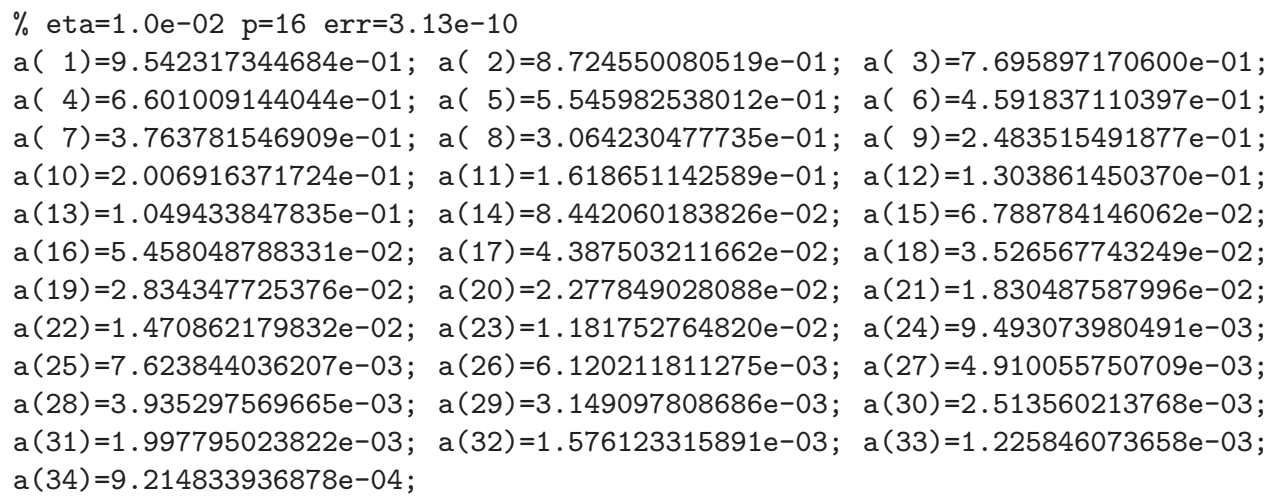

The $\{a(k): k=1, \cdots, 2 P+2\}$ in the table define the cosine set $\left\{c_{j}, \bar{c}_{j}\right\}_{j=0}^{P}$. Indeed, $K^{A}\left(s, \gamma^{2}, T\right)$ depends symmetrically on the cosine parameters. We let the first half of the $a(k)$ define the $c_{j}$, and the second half define the $\bar{c}_{j}$. This table corresponds to $\eta^{-1}=T / \delta=100$, and $|R(s, \boldsymbol{\xi})| \lesssim \varepsilon=3.3 \times 10^{-10}$ (of course $\varepsilon$ here has a different meaning than for Method $1)$. Figure 4 plots the relative pointwise error between $K\left(\xi^{-1} s\right)$ and $K^{A}\left(s, \gamma^{2}, T\right)$ determined by the table. Here we choose $T=20$ and several values of $\xi$. These values correspond to using the approximate boundary condition through a maximum time of 20 with the artificial boundary at $z=\delta=1$.

3.4. Numerical implementation. For our numerical simulations, we work with the homogeneous wave equation in the following FOSH form

$$
\partial_{t} U=-\Pi, \quad \partial_{t} \Pi=-\sum_{i=1}^{3} \partial_{i} \Phi_{i}, \quad \partial_{t} \Phi_{i}=-\partial_{i} \Pi, \quad i=1,2,3,
$$

where the first equation defines $\Pi$ and $\Phi_{i}:=\partial_{i} U$ for $i=1,2,3$. We refer to $\left(U, \Pi, \Phi_{i}\right)$ as the fundamental variables. We stress that in the interior (or "bulk") $0 \leq z \leq \delta$ we evolve the fundamental variables; nevertheless, our above treatment of the boundary conditions at $z=\delta$ has involved the characteristic variables relative to the boundary. Therefore, the full set of variables consists of $\left(U, \Pi, \Phi_{i}\right)$ in the bulk and $\left(W_{j}^{+}, W_{j}^{-}, \Phi_{a, j}\right)$ at $z=\delta$. 


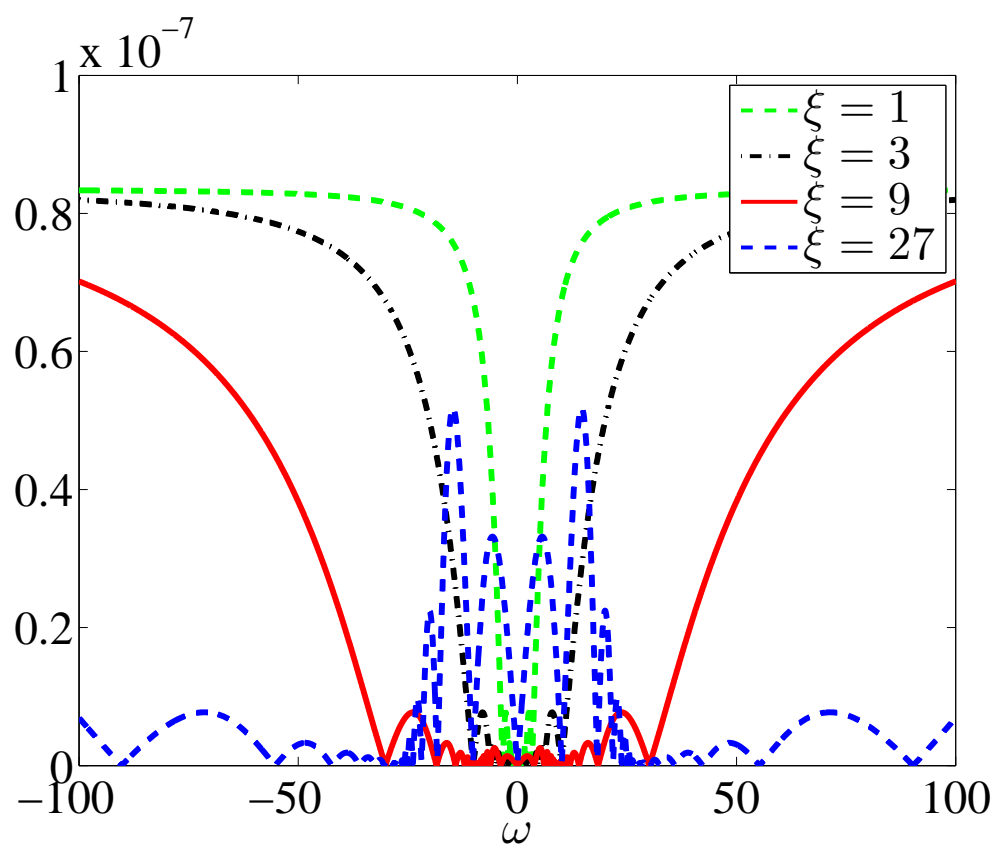

Figure 4. Symbol error $\left|K^{A}\left(s, \gamma^{2}, T\right)-K\left(\xi^{-1} s\right)\right| /\left|K\left(\xi^{-1} s\right)\right|$. Here $s=$ $\mathrm{i} \omega+T^{-1}$, with $T=20$ and $\xi=1,3,9,27$.

We introduce the same Fourier collocation grids $\left\{x_{k}\right\}$ and $\left\{y_{\ell}\right\}$ considered in (16) as well as a Chebyshev-Lobatto grid in the $z$-direction,

$$
\left\{z_{m}=\frac{1}{2}\left(z_{\max }+z_{\min }\right)-\frac{1}{2}\left(z_{\max }-z_{\min }\right) \cos \left(m \pi /\left(N_{z}-1\right)\right): m=0, \ldots, N_{z}-1\right\} .
$$

These grids discretize $\left[x_{\min }, x_{\max }\right] \times\left[y_{\min }, y_{\max }\right] \times\left[z_{\min }, z_{\max }\right]$, and define corresponding nodal values, e.g. $U_{k \ell m} \simeq U\left(x_{k}, y_{\ell}, z_{m}\right)$ and $W_{j, k \ell}^{+} \simeq W_{j}^{+}\left(x_{k}, y_{\ell}\right)$. To compute spatial derivatives of grid functions, which are needed in constructing time derivatives in the method of lines, we use the corresponding collocation derivative matrices: $D_{x}$ (Fourier), $D_{y}$ (Fourier), and $D_{z}$ (Chebyshev-Lobatto); see [14] for the corresponding expressions. Then, for example,

$$
\partial_{z} U\left(x_{k}, y_{\ell}, z_{m}\right) \simeq \sum_{n=0}^{N_{z}-1}\left(D_{z}\right)_{m n} U_{k \ell n},
$$

and similarly for $\partial_{x} U\left(x_{k}, y_{\ell}, z_{m}\right)$ and $\partial_{y} U\left(x_{k}, y_{\ell}, z_{m}\right)$. For example, in the method of lines the $i=1$ case of the last equation in (64) becomes

$$
\partial_{t} \Phi_{1, k \ell m}=-\sum_{h=0}^{N_{x}-1}\left(D_{x}\right)_{k h} \Pi_{h \ell m} .
$$

Evolution (in the bulk) via the classical Runge-Kutta method requires computation of the time-derivatives $\partial_{t} \Pi_{k \ell m}, \partial_{t} \Phi_{i, k \ell m}$, and $\partial_{t} U_{k \ell m}$.

Runge-Kutta evolution of the auxiliary variables on the boundary likewise requires computation of the time-derivatives. Algorithm 1 describes how the system (60) is used to compute the time derivatives of the auxiliary variables. These computations then define the evolution of the boundary system (60). 
Algorithm 1 COMPUte TIME DERIVATIVES OF AUXILIARY VARIABles. Assume the following are given: (i) $\left\{\mathbf{w}_{j}\right\}_{j=0}^{P+1}$ and (ii) $\partial W_{0}^{+} / \partial t$, an inhomogeneous source determined by the interior variables; see Remark 3.6, and below for how this source is determined. The follow steps (which may be performed in any order) compute the required time derivatives.

1: Straightforwardly compute $\left\{\partial_{t} \boldsymbol{\Phi}_{j}\right\}_{j=0}^{P+1}$ using (60c).

2: Given $\partial_{t} W_{0}^{+}$, use (60a) to compute $\partial_{t} W_{j}^{+}$for $j=1, \ldots, P+1$ (i.e. forward iteration).

3: Enforcing the closure condition $W_{P+1}^{-}=0=\partial_{t} W_{P+1}^{-}$, use (60b) to compute $\partial_{t} W_{j}^{-}$for $j=P, \ldots, 0$ (i.e. backward iteration).

The last two paragraphs describes computation of grid-field time derivatives in the bulk and on the boundary; however, the description is missing a key ingredient: implementation of the boundary conditions at $z=0$ and $z=\delta$. We turn to this issue now. Obviously, $\sqrt{2} W^{+}=-\Pi+\Phi_{3}$ and $\sqrt{2} W^{-}=-\Pi-\Phi_{3}$; whence $\Pi-\Phi_{3}$ propagates as $\uparrow$ and $\Pi+\Phi_{3}$ as $\downarrow$, again viewing the $z$-axis as running straight up the page. Before the computation of the bulk time derivatives, boundary values of the bulk variables are overwritten via the following prescriptions. At $z=0$ we overwrite as

$$
\begin{aligned}
\Pi_{k \ell 0} & \leftarrow \frac{1}{2}\left[\left(\Pi_{k \ell 0}+f_{k \ell}\right)+\left(\Pi_{k \ell 0}+\Phi_{3, k \ell 0}\right)\right]=\Pi_{k \ell 0}+\frac{1}{2}\left(\Phi_{3, k \ell 0}+f_{k \ell}\right) \\
\Phi_{3, k \ell 0} & \leftarrow \frac{1}{2}\left[-\left(\Pi_{k \ell 0}+f_{k \ell}\right)+\left(\Pi_{k \ell 0}+\Phi_{3, k \ell 0}\right)\right]=\frac{1}{2}\left(\Phi_{3, k \ell 0}-f_{k \ell}\right) .
\end{aligned}
$$

At $z=\delta$ we overwrite as

$$
\begin{aligned}
\Pi_{k \ell, N_{z}-1} & \leftarrow \frac{1}{2}\left[\left(\Pi_{k \ell, N_{z}-1}-\Phi_{3, k \ell, N_{z}-1}\right)-\sqrt{2} W_{0, k \ell}^{-}\right] \\
\Phi_{3, k \ell, N_{z}-1} & \leftarrow \frac{1}{2}\left[\left(-\Pi_{k \ell, N_{z}-1}+\Phi_{3, k \ell, N_{z}-1}\right)-\sqrt{2} W_{0, k \ell}^{-}\right] .
\end{aligned}
$$

The overwrites $(69 \mathrm{a}, \mathrm{b})$ involve the auxiliary variable $W_{0, k \ell}^{-}$. Likewise, before computing the time derivatives of the auxiliary variables at $z=\delta$, we prescribe

$$
\left(\partial_{t} W_{0}^{+}\right)_{k \ell}=2^{-1 / 2}\left(-\partial_{t} \Pi_{k \ell, N_{z}-1}+\partial_{t} \Phi_{3, k \ell, N_{z}-1}\right) .
$$

The preceding overwrites and prescription, following by straightforward computation of gridfield time derivatives (both for bulk and auxiliary boundary variables) then defines the Runge-Kutta evolution.

3.5. Numerical tests. To test Method 2, we again use (14) and the scenario described in Section 1.2.2. All simulations now also take $N_{z}=10$ and $\delta=0.1$ as the "slab" thickness. As seen from the spatial Fourier transform in $(14),|\widehat{f}(t, \boldsymbol{\xi})| \lesssim 2.1 \times 10^{-16}$ for $\xi \gtrsim 26$. Therefore, the smallest wavelength needed to resolve the source $f(t, \cdot)$ to double precision is $\lambda \simeq 0.242$. We have chosen $\delta$ slightly smaller than this value, with enough points to resolve this wavelength. Using the implementation described in the last subsection, we have generated numerical solutions $F_{k \ell}^{N, P}$ corresponding to $N_{x}=N=N_{y}$ for $N=60,68,76, \ldots, 212$. Each such solution $F_{k \ell}^{N, P}$ is defined on the double Fourier collocation grid (16). To indicate dependence on the radiation boundary conditions, $F_{k \ell}^{N, P}$ also carries a superscript $P$, the chosen number of boundary auxiliary variables defined at $z=\delta$. In all cases we have also 
assumed $\eta=10^{-2}$ in the definition of the radiation boundary conditions; see above. Errors are again computed with (18) and (19).

For each simulation the timestep $\Delta t$ has been chosen both to ensure stability and a spatial error which dominates the temporal error. In practice, $\Delta t=5 /(k N)$, with $k=5$ for $P=4$ and $P=8, k=7$ for $P=12$, and $k=17$ for $P=16$. Figure 5 shows that initially the errors decrease spectrally with increasing $N$, as expected for a Fourier collocation method. However, the errors then saturate at a floor determined by $P$. In each case the floor is consistent with the table errors from faculty. smu.edu/thagstrom/Optimal_cosines.txt, namely the reported size of the reflection coefficient. For $\eta=10^{-2}$ the table errors for $P=$ $4,8,12,16$ are respectively $5.60 \mathrm{e}-04,3.75 \mathrm{e}-06,3.18 \mathrm{e}-08,3.13 \mathrm{e}-10$. The value $3.13 \mathrm{e}-10$ is seen above in the included $\eta=10^{-2}, P=16$ table. Table 2 lists maximum errors and CPU times for select simulations. For each $P=4,8,12,16$ the chosen $N$ corresponds to a point on the curve in Fig. 5 just after the error has saturated.

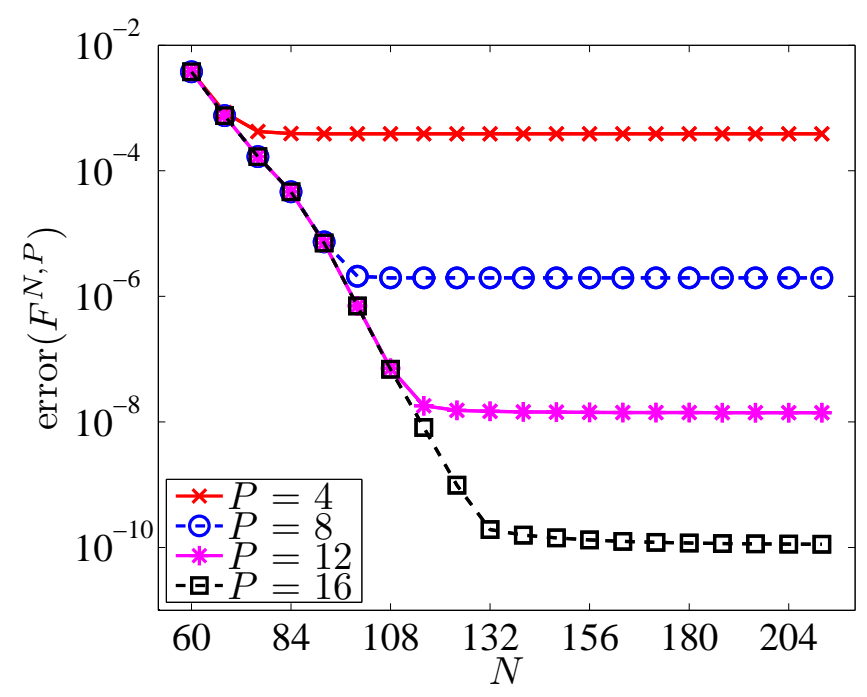

Figure 5. $\operatorname{Error}\left(F^{N, P}\right)$ Computed via (18).

\begin{tabular}{|r|c|c|c|}
\hline$N$ & $P$ & error $_{\infty}\left(F^{N, P}\right)$ & time $(\mathrm{sec})$ \\
\hline 84 & 4 & $1.0050 \mathrm{e}-04$ & $1.6435 \mathrm{E}+02$ \\
108 & 8 & $5.1907 \mathrm{e}-07$ & $7.6091 \mathrm{E}+02$ \\
132 & 12 & $3.6437 \mathrm{e}-09$ & $3.1454 \mathrm{E}+03$ \\
156 & 16 & $4.2889 \mathrm{e}-11$ & $1.7963 \mathrm{E}+04$ \\
\hline
\end{tabular}

Table 2. Maximum errors and CPU times For $\eta=10^{-2}$ Tables.

\section{Conclusions}

We conclude with discussions on the following three issues: (i) comparison of our two methods, (ii) obstacles to implementation in the VM3@A code, and (iii) a possible third method for fast evaluation of the integrals we have considered. 
The results in Tables 1 and 2 indicate that Method 1 is faster than Method 2 for our model scenario. This observation is not unexpected. Indeed, a $t \rightarrow t+\Delta t$ update of $F_{k \ell}(t) \simeq F\left(t, x_{k}, y_{\ell}\right)$ at $N^{2}$ spatial locations formally $\operatorname{costs}^{3} O\left(N^{2}(\log N+d)\right)$ for Method 1 , with the experimental evidence in Appendix A suggesting that $d=O(\log (1 / \varepsilon))$. Here the $O\left(N^{2} \log N\right)$ is the asymptotic complexity of the double FFT, although the relatively small- $N$ values in our experiments have not taken full advantage of the FFT. For Method 2 a $t \rightarrow t+\Delta t$ update of all nodal values costs $O\left(N^{2}\left(N N_{z}+N_{z}^{2}+N P\right)\right)$. Here the first two terms reflect the cost of computing "bulk" derivatives, whereas the last term reflects boundary work. For a unit wave speed $c=1$, Hagstrom and Warburton [6] argue that

$$
P=O\left(\log \frac{1}{\varepsilon} \cdot \log \frac{T}{\delta}\right)
$$

where now $\varepsilon$ (different than the $\varepsilon$ from Method 1) is a bound on the magnitude of the reflection coefficient up to time $T>t_{\mathrm{f}}$ (also different than the $T>t_{\mathrm{f}} \xi_{\max }$ from Method 1). Nevertheless, Method 2 allows for further reduction of the tangential directions. Such reduction would introduce new computational boundaries, with the associated radiation boundary conditions again implemented through the use of auxiliary variables. The key challenge is then to enact the edge and corner "compatibility conditions" at the intersections of the planar boundaries. Implementation of such compatibility conditions is involved, but with such domain reduction in all three spatial directions Method 2 would likely be superior to Method 1. Without truncation in the tangential directions, longer time simulations require larger spatial domains, as discussed earlier. While such enlargement is inelegant, when coupled with Method 1 it may still yield savings.

The above complexity statements should be compared to the direct cost of evaluating the retarded time integral. For late $t$ a naive computation of $F_{k \ell}(t)$ at $N^{2}$ locations costs $O\left(N^{4}\right)$, viewing each of the $N^{2}$ integrations as costing $O\left(N^{2}\right)$ work. However, this cost is mitigated by judicious integration in VM3@A. Indeed, as seen in Fig. 1, the integration is typically over a small portion of the backward cone (see further comments below). Nonetheless, the direct integration must be performed afresh at each timestep. As stated in the introduction, one motivation for this work is the desire to speed up our VM3@A code in which retarded time integration akin to (2) plays a central role. Currently, VM3@A adopts a parallel plate model of the vacuum chamber which entails a shielding boundary condition. Our $J_{0}$ approach (Method 1 ) is associated with the case of no shielding and (2) as the retarded time integration; we will explore this case first. By our estimates, we need an approximation to $J_{0}(t)$ which is accurate to $10^{-3}$ over $t$-values up to $T=10^{6}$; our current approximations far surpass these modest requirements. Although doing so would have changed little, our estimates have not taken advantage of the small source (bunch) support $\mathcal{S}_{f}(t)$. Again, by the source we mean $f(t, \mathbf{x})$ in (2), corresponding to the electron bunch in the physical scenario. Nevertheless, taking advantage of the small $f$-support proves useful in VM3@A. Indeed, in VM3@A applications to date, the electromagnetic fields, corresponding to $F(t, \mathbf{x})$ in $(2)$, only need to be evaluated at spatial points $\mathbf{x}$ inside the bunch, whereas the $J_{0}$ approach requires evaluation of $F(t, \mathbf{x})$ on its own full support $\mathcal{S}_{F}(t)$ in order to work with its Fourier transform. Thus, the speed up in the calculation of (2) afforded by the $J_{0}$ approach will be counterbalanced by the need for a larger spatial domain. However, we note that calculation of the full support of the

\footnotetext{
${ }^{3}$ This complexity scaling improves on the naive scaling stated after Eq. (7) in Ref. [4].
} 
fields ( $F$-support) is important for some applications (e.g., in an actual experiment where the fields are measured outside of the bunch). In addition, we plan to consider the shielding condition, and we are investigating the integrals analogous to (2) which arise from the image charges needed to incorporate this shielding. Furthermore, we plan to incorporate more realistic vacuum chamber boundary conditions in VM3@A. Here our second approach (Method 2) may be needed.

Finally, following a suggestion of T. Hagstrom, we sketch a third method for evaluation the integral (1), one that we have not explored. Another exact radiation boundary condition at $z=\delta$ is $[15,16]$

$$
s \stackrel{v}{U}(s, \boldsymbol{\xi}, \delta)+\partial_{z} \stackrel{v}{U}(s, \boldsymbol{\xi}, \delta)=\left(-\sqrt{s^{2}+\xi^{2}}+s\right) \stackrel{v}{U}(s, \boldsymbol{\xi}, \delta) .
$$

This formula is consistent with the form (59) of the solution above the sheet. Moreover, the explicit form for $\stackrel{v}{U}(s, \boldsymbol{\xi}, z)$ given in (59) shows that the $\delta \rightarrow 0^{+}$limit of (72) is

$$
s \stackrel{v}{U}(s, \boldsymbol{\xi}, 0)=\left(-\sqrt{s^{2}+\xi^{2}}+s\right) \stackrel{v}{U}(s, \boldsymbol{\xi}, 0)+\stackrel{\vee}{f}(s, \boldsymbol{\xi}) .
$$

We then consider a sum-of-poles approximation $-\sqrt{s^{2}+\xi^{2}}+s \simeq \sum_{k=1}^{d} \alpha_{k} /\left(s-\beta_{k}\right)$ of the plane kernel, and define $\Theta(t, \boldsymbol{\xi})=\sum_{k=1}^{d} \alpha_{k} \Theta_{k}(t, \boldsymbol{\xi})$, where

$$
\Theta_{k}(t, \boldsymbol{\xi})=\int_{0}^{t} e^{\beta_{k}\left(t-t^{\prime}\right)} \widehat{U}\left(t^{\prime}, \boldsymbol{\xi}, 0\right) d t^{\prime} .
$$

The third method would involve evolution of the approximate system

$$
\partial_{t} \widehat{U}(t, \boldsymbol{\xi}, 0)=\Theta(t, \boldsymbol{\xi})+\widehat{f}(t, \boldsymbol{\xi}), \quad \partial_{t} \Theta_{k}(t, \boldsymbol{\xi})=\beta_{k} \Theta_{k}(t, \boldsymbol{\xi})+\widehat{U}(t, \boldsymbol{\xi}, 0) \text { for } k=1, \ldots, d .
$$

We believe that an implementation of this method would perform similar to our first method.

\section{ACKNOWLEDGMENTS}

All authors acknowledge support through DOE grant DE-FG-99ER41104. SRL and DAB also gratefully acknowledge support through NSF grant PHY 0855678. The software for approximating $J_{0}(t)$ as a sum of exponentials was developed under that support. We thank Daniel Appelö and Tom Hagstrom for comments, and particularly thank Gabriele Bassi for both discussions and preliminary calculations with VM3@A.

\section{Appendix A. Tables for approximation of $J_{0}(t)$}

Table 3 specifies one approximation $A(t)$ of $J_{0}(t)$ as a sum of poles. This table corresponds to $d_{\text {pair }}=55$ and $\eta=10^{-6}$, that is $T=10^{6}$. The format in this table is as follows. The 55 lines list $j, m_{j}^{R}, m_{j}^{I}, k_{j}^{R}, k_{j}^{I}$ for $j=1, \ldots, 55$. These numbers specify the approximate kernel

$$
A(t)=\sum_{j=1}^{d_{\text {pair }}}\left(m_{j} e^{k_{j} t}+\bar{m}_{j} e^{\bar{k}_{j} t}\right), \quad m_{j}:=m_{j}^{R}+\mathrm{i} m_{j}^{I}, \quad k_{j}:=k_{j}^{R}+\mathrm{i} k_{j}^{I} .
$$

The pole locations $k_{j}$ (blue) and $\bar{k}_{j}$ (red) are shown in Fig. 6. Figures 7 and 8 demonstrate that this $A(t)$ accurately approximates $J_{0}(t)$ for $t \lesssim 10^{6}$. In these figures we normalize the pointwise error by $\max \{1, \sqrt{2 /(\pi t)}\}$, that is the asymptotic amplitude of $J_{0}(t)$ for $t \gtrsim 0.6366$. 


\begin{tabular}{|c|c|c|c|c|}
\hline 1 & $6.34553435678094553 \mathrm{E}-02$ & $-4.52057031527512725 \mathrm{E}-03$ & $-9.84157254021301103 \mathrm{E}-01$ & $1.40682304911772850 \mathrm{E}-01$ \\
\hline 2 & $5.98095983170248993 \mathrm{E}-02$ & $-1.25960477775790956 \mathrm{E}-02$ & $-9.10002737746297939 \mathrm{E}-01$ & $4.00755812865457362 \mathrm{E}-01$ \\
\hline 3 & $5.37193217377211789 \mathrm{E}-02$ & $-1.83310409901512175 \mathrm{E}-02$ & $-7.86871563981832596 \mathrm{E}-01$ & $6.08562486251849243 \mathrm{E}-01$ \\
\hline 4 & $4.67116371056696507 \mathrm{E}-02$ & $-2.14450268225980361 \mathrm{E}-02$ & $-6.47095056403736290 \mathrm{E}-01$ & $7.56324532715444916 \mathrm{E}-01$ \\
\hline 5 & $3.98962552980207310 \mathrm{E}-02$ & $-2.24201982100573368 \mathrm{E}-02$ & $-5.14376139188261027 \mathrm{E}-01$ & $8.53039181915166747 \mathrm{E}-01$ \\
\hline 6 & 3. 38156815695398526E-02 & $-2.19554634593517353 \mathrm{E}-02$ & $-4.00198368121055924 E-01$ & $9.13032025641817086 \mathrm{E}-01$ \\
\hline 7 & $2.86198096185465922 \mathrm{E}-02$ & $-2.06536515281793709 \mathrm{E}-02$ & $-3.07356734923560435 E-01$ & $9.49055970311817835 \mathrm{E}-01$ \\
\hline 8 & $2.42642587542517908 \mathrm{E}-02$ & $-1.89391515462693067 \mathrm{E}-02$ & $-2.34268735581378003 E-01$ & $9.70291158553201827 \mathrm{E}-01$ \\
\hline 9 & $2.06350579096597693 \mathrm{E}-02$ & $-1.70797764659753721 \mathrm{E}-02$ & $-1.77788339082533946 \mathrm{E}-01$ & $9.82688487881354769 \mathrm{E}-01$ \\
\hline 10 & $1.76085846605725807 \mathrm{E}-02$ & $-1.52322985621904133 \mathrm{E}-02$ & $-1.34600733906033518 \mathrm{E}-01$ & $9.89895977448459004 \mathrm{E}-01$ \\
\hline 11 & $1.50745579200699218 \mathrm{E}-02$ & $-1.34819557270006871 \mathrm{E}-02$ & $-1.01774322805270176 \mathrm{E}-01$ & $9.94083088083540330 \mathrm{E}-01$ \\
\hline 12 & $1.29418326657610562 \mathrm{E}-02$ & $-1.18702388924038714 \mathrm{E}-02$ & $-7.69064433008709701 \mathrm{E}-02$ & $9.96519165720732649 \mathrm{E}-01$ \\
\hline 13 & 1. $11374184007151383 \mathrm{E}-02$ & $-1.04128154237031093 \mathrm{E}-02$ & $-5.81016799739324220 \mathrm{E}-02$ & $9.97940805133465572 \mathrm{E}-01$ \\
\hline 14 & $9.60344911915840500 \mathrm{E}-03$ & $-9.11062353106553919 \mathrm{E}-03$ & $-4.38949356303393210 \mathrm{E}-02$ & $9.98773945878555656 \mathrm{E}-01$ \\
\hline 15 & $8.29399490726871141 \mathrm{E}-03$ & $-7.95659253963650024 \mathrm{E}-03$ & $-3.31663571621436345 \mathrm{E}-02$ & 9.99264733476774 \\
\hline 16 & $7.17234143111895506 \mathrm{E}-03$ & $-6.93965511087873383 \mathrm{E}-03$ & $-2.50653040840208025 \mathrm{E}-02$ & $9.99555588216363029 \mathrm{E}-01$ \\
\hline 17 & $6.20885243757796810 \mathrm{E}-03$ & $-6.04710685954324944 \mathrm{E}-03$ & $-1.89478663145966179 \mathrm{E}-02$ & $9.99729124459884778 \mathrm{E}-01$ \\
\hline 18 & $5.37935065504614016 \mathrm{E}-03$ & $-5.26596399415416130 \mathrm{E}-03$ & $-1.43275438983517743 \mathrm{E}-02$ & $9.99833434961792353 \mathrm{E}-01$ \\
\hline 19 & $4.66390915030450231 \mathrm{E}-03$ & $-4.58371592085338878 \mathrm{E}-03$ & $-1.08371281690762813 \mathrm{E}-02$ & $9.99896639396619081 \mathrm{E}-01$ \\
\hline 20 & $4.04595124330314022 \mathrm{E}-03$ & $-3.98871407619327033 \mathrm{E}-03$ & $-8.19957736567113332 \mathrm{E}-03$ & $9.99935264082766777 \mathrm{E}-01$ \\
\hline 21 & $3.51157635036178895 \mathrm{E}-03$ & $-3.47034417426211063 \mathrm{E}-03$ & $-6.20590649189851424 \mathrm{E}-03$ & $9.99959078841135194 \mathrm{E}-01$ \\
\hline 22 & $3.04904935110888775 \mathrm{E}-03$ & $-3.01907179358642734 \mathrm{E}-03$ & $-4.69846219333353819 \mathrm{E}-03$ & $9.99973897149130566 \mathrm{E}-01$ \\
\hline 23 & $2.64840841347512734 \mathrm{E}-03$ & $-2.62641598886491699 \mathrm{E}-03$ & $-3.55829620181412574 \mathrm{E}-03$ & $9.99983203178642120 \mathrm{E}-01$ \\
\hline 24 & $2.30115927664418557 \mathrm{E}-03$ & $-2.28488437582584712 \mathrm{E}-03$ & $-2.69564305122719809 \mathrm{E}-03$ & $9.99989101349984356 \mathrm{E}-01$ \\
\hline 25 & $2.00003322775159264 \mathrm{E}-03$ & $-1.98789012956364936 \mathrm{E}-03$ & $-2.04274251817353323 \mathrm{E}-03$ & $9.99992873290697800 \mathrm{E}-01$ \\
\hline 26 & $1.73879272102273586 \mathrm{E}-03$ & $-1.72966292542240431 \mathrm{E}-03$ & $-1.54843041186466055 \mathrm{E}-03$ & $9.99995306354049084 \mathrm{E}-01$ \\
\hline 27 & $1.51207320575300895 \mathrm{E}-03$ & $-1.50516063720447059 \mathrm{E}-03$ & $-1.17406199238230060 \mathrm{E}-03$ & 9.99996888615788 \\
\hline 28 & $1.31525282506717788 \mathrm{E}-03$ & $-1.30998562270017627 \mathrm{E}-03$ & $-8.90439318906363501 \mathrm{E}-04$ & 9.9999792 \\
\hline 29 & $1.14434400055449905 \mathrm{E}-03$ & $-1.14030756408797849 \mathrm{E}-03$ & $-6.75494828630796717 \mathrm{E}-04$ & $9.99998609559613619 \mathrm{E}-01$ \\
\hline 30 & $9.95902578716524687 \mathrm{E}-04$ & $-9.92793598674134262 \mathrm{E}-04$ & $-5.12544586231112850 \mathrm{E}-04$ & $9.99999063856270817 \mathrm{E}-01$ \\
\hline 31 & $8.66951202356933330 \mathrm{E}-04$ & $-8.64545836976552150 \mathrm{E}-04$ & $-3.88970732969750329 \mathrm{E}-04$ & $9.99999367247550006 \mathrm{E}-01$ \\
\hline 32 & $7.54914282347560260 \mathrm{E}-04$ & $-7.53045978733906621 \mathrm{E}-04$ & $-2.95227376765217073 \mathrm{E}-04$ & $9.99999570886057160 \mathrm{E}-01$ \\
\hline 33 & $6.57562574291989774 \mathrm{E}-04$ & $-6.56106475676115122 \mathrm{E}-04$ & $-2.24090291757568200 \mathrm{E}-04$ & $9.99999708177516578 \mathrm{E}-01$ \\
\hline 34 & $5.72965804195971715 \mathrm{E}-04$ & $-5.71827653219207136 \mathrm{E}-04$ & $-1.70090453296295140 \mathrm{E}-04$ & $9.99999801095022079 \mathrm{E}-01$ \\
\hline 35 & $4.99452059208945463 \mathrm{E}-04$ & $-4.98560233526098208 E-04$ & $-1.29086226641440665 \mathrm{E}-04$ & $9.99999864187758424 \mathrm{E}-01$ \\
\hline 36 & $4.35572912127245838 \mathrm{E}-04$ & $-4.34872669366592234 \mathrm{E}-04$ & $-9.79401641719752780 \mathrm{E}-05$ & $9.99999907147363356 \mathrm{E}-01$ \\
\hline 37 & $3.80073430659571607 \mathrm{E}-04$ & $-3.79522699220714162 \mathrm{E}-04$ & $-7.42747515780101733 \mathrm{E}-05$ & $9.99999936464750115 \mathrm{E}-01$ \\
\hline 38 & $3.31866335919006786 \mathrm{E}-04$ & $-3.31432611038251444 \mathrm{E}-04$ & $-5.62877581052122545 \mathrm{E}-05$ & $9.99999956508444175 \mathrm{E}-01$ \\
\hline 39 & $2.90009712731396035 \mathrm{E}-04$ & $-2.89667767411294775 \mathrm{E}-04$ & $-4.26126009424064273 \mathrm{E}-05$ & $9.99999970231182544 \mathrm{E}-01$ \\
\hline 40 & $2.53687794977007283 \mathrm{E}-04$ & $-2.53417977028711042 \mathrm{E}-04$ & $-3.22127160487908920 \mathrm{E}-05$ & $9.99999979635998210 \mathrm{E}-01$ \\
\hline 41 & $2.22194404180624349 \mathrm{E}-04$ & $-2.21981352436152501 \mathrm{E}-04$ & $-2.43016281457418485 E-05$ & $9.99999986085877677 \mathrm{E}-01$ \\
\hline 42 & $1.94918664187375261 \mathrm{E}-04$ & $-1.94750340571773844 \mathrm{E}-04$ & $-1.82824489556946771 \mathrm{E}-05$ & $9.99999990510686311 \mathrm{E}-01$ \\
\hline 43 & $1.71332681193924235 \mathrm{E}-04$ & $-1.71199637055812955 \mathrm{E}-04$ & $-1.37020686031080858 \mathrm{E}-05$ & $9.99999993546093945 \mathrm{E}-01$ \\
\hline 44 & $1.50980932681381396 \mathrm{E}-04$ & $-1.50875739578563767 \mathrm{E}-04$ & $-1.02164635245701214 \mathrm{E}-05$ & $9.99999995627378025 \mathrm{E}-01$ \\
\hline 45 & $1.33471139391104444 \mathrm{E}-04$ & $-1.33387942976641220 \mathrm{E}-04$ & $-7.56441842046683233 \mathrm{E}-06$ & $9.99999997053060825 \mathrm{E}-01$ \\
\hline 46 & $1.18466429361012505 \mathrm{E}-04$ & $-1.18400600378152262 \mathrm{E}-04$ & $-5.54761997927367308 \mathrm{E}-06$ & $9.99999998028167069 \mathrm{E}-01$ \\
\hline 47 & $1.05678630099742563 \mathrm{E}-04$ & $-1.05626489684061745 E-04$ & $-4.01557899113664002 E-06$ & $9.99999998693681147 \mathrm{E}-01$ \\
\hline 48 & $9.48625391605787604 \mathrm{E}-05$ & $-9.48211471540738154 \mathrm{E}-05$ & $-2.85421465825561657 E-06$ & $9.99999999146628251 \mathrm{E}-01$ \\
\hline 49 & $8.58110517503656342 \mathrm{E}-05$ & $-8.57780501461675958 \mathrm{E}-05$ & $-1.97722021833143886 \mathrm{E}-06$ & $9.99999999453751584 \mathrm{E}-01$ \\
\hline 50 & $7.83510580330856655 \mathrm{E}-05$ & $-7.83245492921476329 \mathrm{E}-05$ & $-1.31954502681241266 \mathrm{E}-06$ & $9.99999999660864144 \mathrm{E}-01$ \\
\hline 51 & $7.23400330508021901 \mathrm{E}-05$ & $-7.23184759174249886 \mathrm{E}-05$ & $-8.32492218265339394 \mathrm{E}-07$ & 9.99999999799299354E-01 \\
\hline 52 & $6.76632391192637045 \mathrm{E}-05$ & $-6.76453611127359805 \mathrm{E}-05$ & $-4.80056029113333401 \mathrm{E}-07$ & $9.99999999890350617 \mathrm{E}-01$ \\
\hline 53 & $6.42314744785599074 \mathrm{E}-05$ & $-6.42162005817868219 \mathrm{E}-05$ & $-2.36218563560535326 \mathrm{E}-07$ & $9.99999999948284385 \mathrm{E}-01$ \\
\hline 54 & $6.19793230216314057 \mathrm{E}-05$ & $-6.19657150766521923 E-05$ & $-8.29995782654961473 E-08$ & $9.99999999982352588 \mathrm{E}-01$ \\
\hline 55 & $6.08638718748625530 \mathrm{E}-05$ & $-6.08510766366933731 \mathrm{E}-05$ & $-9.11055835096548994 \mathrm{E}-09$ & $9.99999999998091642 \mathrm{E}-01$ \\
\hline
\end{tabular}

Table 3 . TABle SPecifying an approximation $A(t)$ To $J_{0}(t)$. The text explains the relationship between the entries and the sum-of-exponentials $A(t)$. 


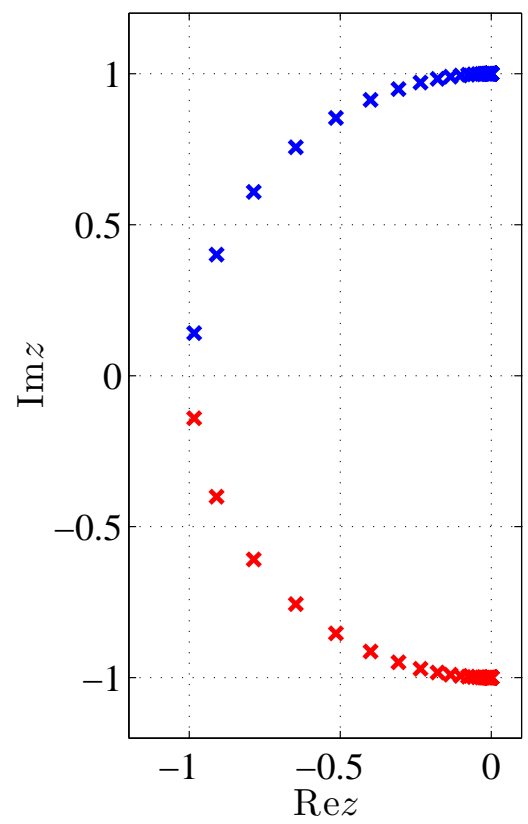

Figure 6. Pole locations for Table 3.
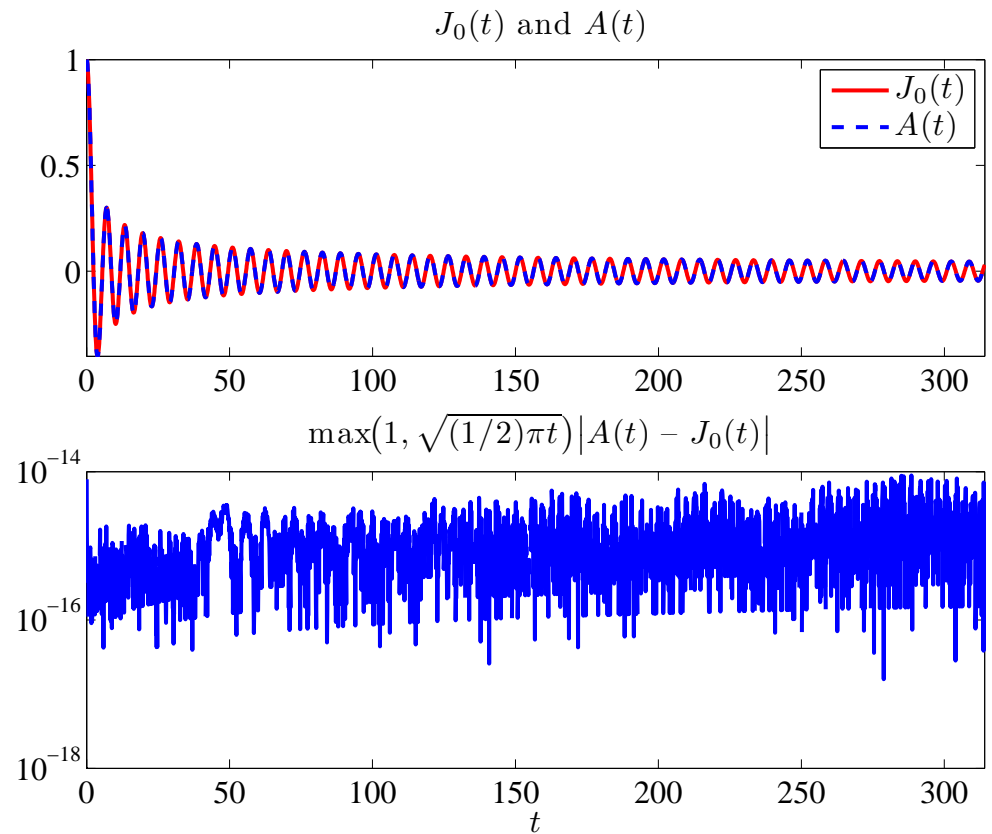

Figure 7. ERROR FOR TABLE 3 NEAR $10^{0}$.

Table 4 collects results for other sum-of-poles $\widetilde{A}(s)$ and sum-of-exponentials $A(t)$ kernels that we have constructed. Here the quoted error tolerances $\varepsilon$ may be interpreted as either 


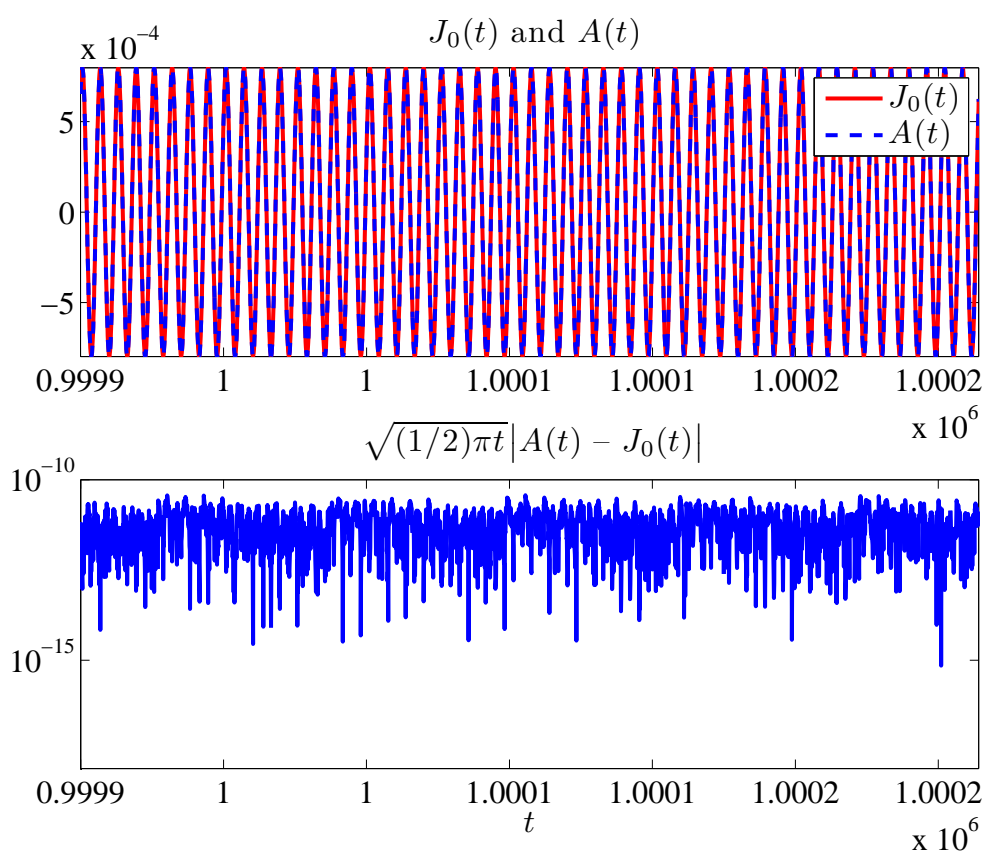

Figure 8. ERror for TABle 3 NeAR $10^{6}$.

\begin{tabular}{|l|l|}
\hline \multicolumn{2}{|c|}{$T=10^{4}$} \\
\hline$d$ & $\varepsilon$ \\
36 & $10^{-6}$ \\
46 & $10^{-8}$ \\
56 & $10^{-10}$ \\
66 & $10^{-12}$ \\
\hline
\end{tabular}

\begin{tabular}{|l|l|}
\hline \multicolumn{2}{|c|}{$T=10^{6}$} \\
\hline$d$ & $\varepsilon$ \\
50 & $10^{-6}$ \\
66 & $10^{-8}$ \\
82 & $10^{-10}$ \\
\hline
\end{tabular}

\begin{tabular}{|l|l|}
\hline \multicolumn{2}{|c|}{$T=10^{8}$} \\
\hline$d$ & $\varepsilon$ \\
66 & $10^{-6}$ \\
86 & $10^{-8}$ \\
\hline
\end{tabular}

TABle 4. TOlERANCES FOR SUM-OF-POles/EXPONEntial APPROXimations.

of the following control statements:

$$
\sup _{y \in \mathbb{R}} \frac{\left|\widetilde{A}\left(T^{-1}+\mathrm{i} y\right)-\widetilde{J}_{0}\left(T^{-1}+\mathrm{i} y\right)\right|}{\left|\widetilde{J}_{0}\left(T^{-1}+\mathrm{i} y\right)\right|}<\varepsilon, \quad \sup _{t \in[0, T]} \max (1, \sqrt{(1 / 2) \pi t})\left|A(t)-J_{0}(t)\right|<\varepsilon .
$$

Note that under the $T=10^{6}$ results in Table 4 , the case of our 110 pole approximation specified in Table 3 is not listed. Over $t \in\left[0, T=10^{6}\right]$ the 110 pole table has a uniform accuracy which is essentially the same as an 82 pole table; however, it is quite a bit more accurate near $t=0$. For each of the chosen values of $T$, the largest $d$ value listed as a result in Table 4 roughly corresponds to where the error saturates. The effect corresponds to an inevitable breakdown tied to our double precision representation of the poles and residues which specify each table. We stress that we have obtained each approximation (through the procedure described in the next appendix) using only quadruple precision calculations. However, we typically store tables in a double precision format. To examine the effect of the storage format, Fig. 9 plots scaled pointwise errors between $A(t)$ and $J_{0}(t)$ over $\left[1, T=10^{8}\right]$ for both of the approximations $A(t)$ listed under $T=10^{8}$ in Table 4 , in addition to the corresponding errors for three additional approximations involving more exponentials (the $\varepsilon$ 
for which are not listed in Table 4). For the kernels with more terms, we see that the accuracy degrades at larger times, and uniform accuracy cannot be maintained over $\left[0, T=10^{8}\right]$ for $\varepsilon \lesssim 10^{-8}$. However, as indicated in Fig. 10, by switching to a quadruple representation of the poles and residues which specify each kernel (and of course computing the errors in quadruple precision), we recover uniform accuracies. Tables will be available at [17].

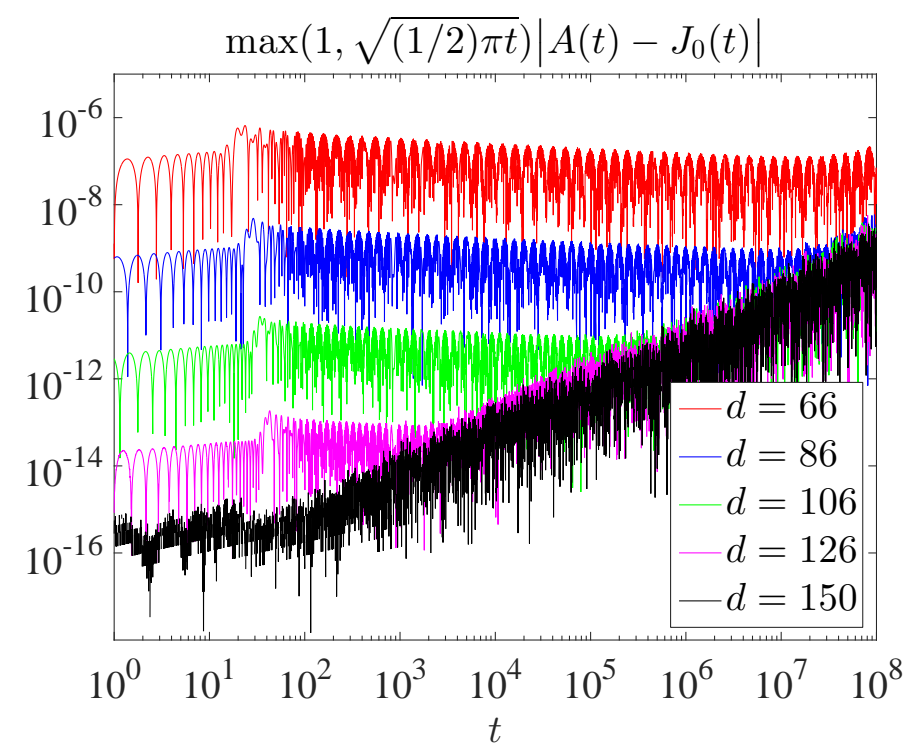

Figure 9. ERROR RESUlts FOR TABLES IN DOUBLE PRECISION FORMAT.

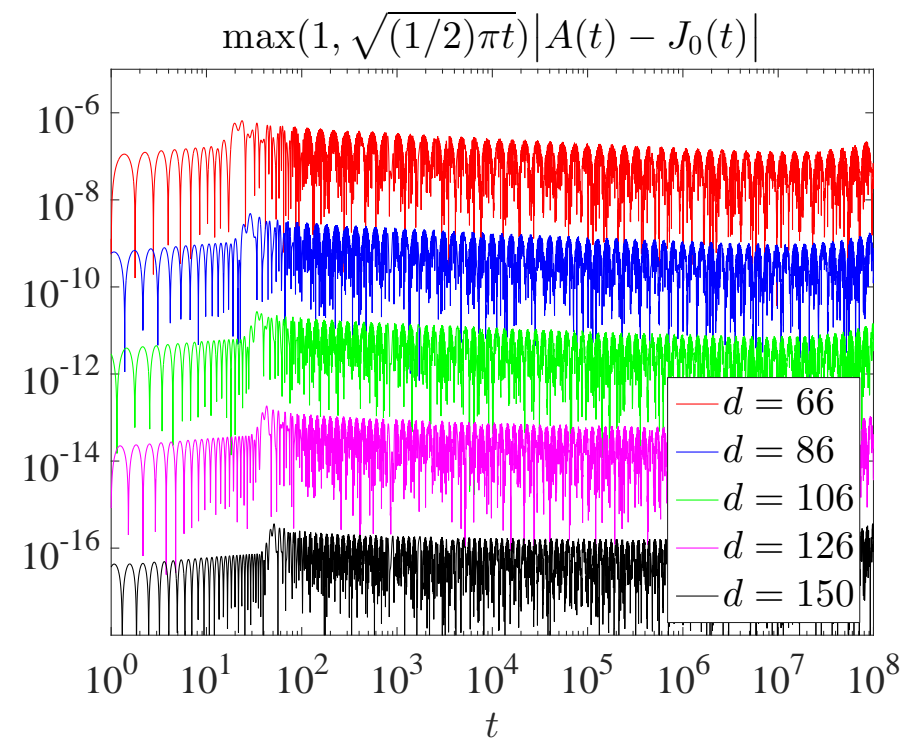

Figure 10. ERror RESUlts FOR TABLES IN QUADRUPLE PRECISION FORMAT. 


\section{Appendix B. AGH COMPRESSION ALGORITHM}

This appendix discusses Alpert-Greengard-Hagstrom "kernel compression" [7, 9, 18, 10]. We only remark on differences between our version of algorithm and Jiang and Xu's [9, 10].

B.1. Overview. Our goal is to solve the following series of minimization problems

$$
\min _{P^{(k+1)}, Q^{(k+1)}} \int_{-\infty}^{\infty}\left|\frac{P^{(k+1)}(x+\mathrm{i} y)}{Q^{(k)}(x+\mathrm{i} y)}-\frac{Q^{(k+1)}(x+\mathrm{i} y)}{Q^{(k)}(x+\mathrm{i} y)} \widetilde{J}_{0}(x+\mathrm{i} y)\right|^{2} \mathrm{~d} y,
$$

where for each $k$ the task is to find polynomials $P^{(k+1)}(s)$ and $Q^{(k+1)}(s)$, with $d=\operatorname{deg}\left(Q^{(k)}\right)=$ $\operatorname{deg}\left(Q^{(k+1)}\right)=\operatorname{deg}\left(P^{(k+1)}\right)+1$. Here, $\widetilde{J}_{0}(s)=\left(s^{2}+1\right)^{-1 / 2}$, where $s=x+\mathrm{i} y$, and $x$ is the parameter $T^{-1}$ discussed earlier. Do not confuse the $x=\operatorname{Re} s, y=\operatorname{Im} s$ here with the physical $x, y$ coordinates used earlier. Assuming that $Q^{(k)}(s)$ is known, the AGH algorithm solves (78) via an ingenious use of the Gram-Schmidt process; the corresponding inner product is

$$
\langle h, g\rangle_{k}=\int_{-\infty}^{\infty} h(y) \bar{g}(y) m_{k}(y) \mathrm{d} y, \quad m_{k}(y)=1 /\left|Q^{(k)}(x+\mathrm{i} y)\right|^{2} .
$$

To start, we need the $d$ roots of $Q^{(0)}(s)$. We place these on the semi-circle in the left-half $s$-plane from $s=-$ i to $s=\mathrm{i}$. To express the final rational function as a pole sum

$$
\frac{P^{\left(k_{\max }\right)}(s)}{Q^{\left(k_{\max }\right)}(s)}=: \frac{P(s)}{Q(s)}=\sum_{n=1}^{d} \frac{\gamma_{n}}{s-\beta_{n}}, \quad k_{\max } \gg 1,
$$

we must know how to evaluate $P(s)$ and $Q(s)$ and their derivatives at any $s$. Moreover, for each $k$ we must perform such an evaluation to determine $m_{k}(y)$. See [18] for the procedure.

B.2. Numerical inner product. This subsection touches on how we have implemented (79). First, we choose a finite integration window $\left[-y_{\max }, y_{\max }\right]$, typically with $y_{\max }=10$. We then employ an adaptive trapezoid rule. To obtain the rule, we first follow Jiang [9] and $\mathrm{Xu}$ and Jiang [10] and construct a binary tree of subintervals (described below). These subintervals then define both an adaptive grid $\left\{y_{j}\right\}_{j=0}^{2 Q N_{\text {int }}}$ and corresponding integration weights $\left\{\mu_{j}\right\}_{j=0}^{2 Q N_{\text {int }}}$ for positive integers $Q$ and $N_{\text {int }}$. Do not confuse the $\mu_{j}$ with the $k$ th weight function $m_{k}(y)$. Using the $y$-grid and $\mu$-weights, we define a discrete version $\langle,\rangle_{k}^{\mathrm{d}}$ of $(79)$ :

$$
\langle h, g\rangle_{k}^{\mathrm{d}}=\sum_{j=0}^{2 Q N_{\mathrm{int}}} h\left(y_{j}\right) \bar{g}\left(y_{j}\right) m_{k}\left(y_{j}\right) \mu_{j} .
$$

In our case the real and imaginary parts of each function are respectively of even and odd $y$ parity; we enforce this in (81). With (81), we solve (in quadruple precision) the least squares problem via the Gram-Schmidt procedure mentioned above, crucially with reorthogonalization at each step. We use the binary tree from $[9,10]$, but not recursive compression with a "near pole" criterion. Instead, we use the tree to construct the global y-grid and $\mu$-weights.

The Jiang-Xu binary tree starts with $\left[0, y_{\max }\right]$ at tree-height $H=0$ and defines a sequence

$$
\left[0, b_{1}\right]=\left[a_{1}, b_{1}\right],\left[a_{2}, b_{2}\right], \ldots\left[a_{N_{\mathrm{int}}}, b_{N_{\mathrm{int}}}\right]=\left[a_{N_{\mathrm{int}}}, y_{\mathrm{max}}\right],
$$

of adjacent subintervals. On each subinterval $f(y):=\widetilde{J}_{0}(x+\mathrm{i} y)$ is slowly varying to a prescribed tolerance. "Slowly varying" means that on a given subinterval, a Chebyshev series for $f(y)$ features a rapidly decaying spectrum. Figure 11 depicts each subinterval with 
a height corresponding to its tree-height $H$. Small subintervals cluster around $y=1$ where the variation of $f(y)$ is largest. On the non-negative $y$-axis the $y_{j}=y(j)$ and $\mu_{j}=\mu(j)$ are

$$
\begin{aligned}
& \left.\begin{array}{l}
y\left(Q N_{\text {int }}+(j-1) Q+q\right)=a_{j}+q \Delta y_{j} \\
\Delta y_{j}=\left(b_{j}-a_{j}\right) / Q
\end{array}\right\} \text { for } q=0, \cdots, Q \text { and } j=1, \cdots, N_{\text {int }} \\
& \left.\begin{array}{l}
\mu\left(Q N_{\text {int }}+\right. \\
(j-1) Q)=\frac{1}{2}\left[\Delta y_{j}+\left(1-\delta_{j 1}\right) \Delta y_{j-1}\right] \\
\mu\left(Q N_{\text {int }}+(j-1) Q+q\right)=\Delta y_{j} \\
\mu\left(Q N_{\text {int }}+j Q\right)=\frac{1}{2}\left[\Delta y_{j}+\Delta y_{j+1}\right]
\end{array}\right\} \text { for } q=1, \cdots, Q-1 \text { and } j=1, \cdots, N_{\text {int }} .
\end{aligned}
$$

By parity then $y(i)=-y\left(2 Q N_{\text {int }}-i\right)$ and $\mu(i)=\mu\left(2 Q N_{\text {int }}-i\right)$ for $i=0, \ldots, Q N_{\text {int }}-1$.

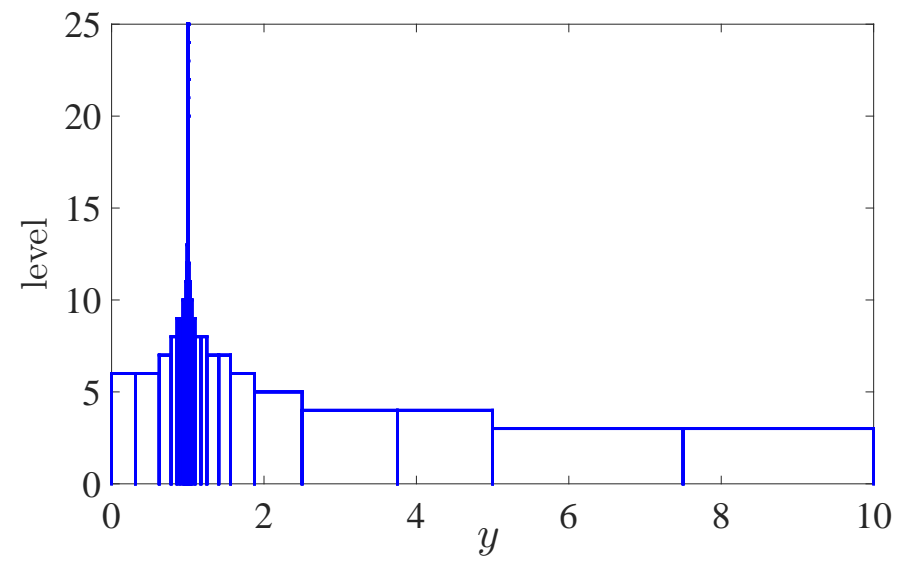

Figure 11. Subintervals $\left[a_{1}, b_{1}\right],\left[a_{2}, b_{2}\right], \ldots\left[a_{N_{\mathrm{int}}}, b_{N_{\mathrm{int}}}\right]$.

\section{Appendix C. Spacetime form of the Boundary CONDition}

Equation (56) from the main text reads

$$
\stackrel{\vee}{W}^{-}(s, \boldsymbol{\xi}, \delta)=-\stackrel{\vee}{W}^{+}(s, \boldsymbol{\xi}, \delta)+\stackrel{\vee}{P}_{1}(s, \boldsymbol{\xi}) \stackrel{\vee}{V}(s, \boldsymbol{\xi}), \quad \stackrel{\vee}{P}_{1}(s, \boldsymbol{\xi})=\frac{2}{\sqrt{s^{2}+\xi^{2}}+s},
$$

where $\stackrel{v}{V}(s, \boldsymbol{\xi}):=s \stackrel{\vee}{W}^{+}(s, \boldsymbol{\xi}, \delta)$. We express Eq. (57) as

$$
W^{-}(t, \mathbf{x}, \delta)=-W^{+}(t, \mathbf{x}, \delta)+\mathcal{I}\left(t, \mathbf{x} ; D_{0} W^{+}(\cdot, \cdot, \delta)\right),
$$

where $V(t, \mathbf{x}):=D_{0} W^{+}(t, \mathbf{x}, \delta)$ and

$$
\mathcal{I}(t, \mathbf{x} ; V):=\frac{1}{\pi} \int_{0}^{t} d t^{\prime} \int_{0}^{1} d \varrho \varrho \int_{0}^{2 \pi} d \theta V\left(t^{\prime}, \mathbf{x}+\left(t-t^{\prime}\right) \varrho \boldsymbol{\nu}(\theta)\right) .
$$

Below we also use the notation $M(s, \boldsymbol{\xi})=\stackrel{\vee}{P}_{1}(s, \boldsymbol{\xi})$. As shown below, $\stackrel{\vee}{P}(s, \boldsymbol{\xi})$ is the FourierLaplace transform of

$$
P_{1}(t, \mathbf{x}):=2 t^{-2} H(t-|\mathbf{x}|),
$$

and another expression for (86) is

$$
\mathcal{I}(t, \mathbf{x} ; V)=\frac{1}{2 \pi} \int_{0}^{t} d t^{\prime} \int_{\mathbb{R}^{2}} d \mathbf{x}^{\prime} P_{1}\left(t-t^{\prime}, \mathbf{x}-\mathbf{x}^{\prime}\right) V\left(t^{\prime}, \mathbf{x}^{\prime}\right) .
$$


This appendix establishes the equivalency between (84) and (85), more precisely the equivalency between $\stackrel{\vee}{P}{ }_{1}^{V}$ and $\mathcal{I}$.

C.1. Overview and assumptions. Now, $V=D_{0} W^{+}(\cdot, \cdot, \delta)$ for the specific case of Eq. (57), but this appendix views $V$ as a generic function, although one subject to further assumptions:

(A1) $V$ is continuous in all its independent variables, except possibly in $t$ at $t=0$. Moreover, $V(t, \mathbf{x})=0$ for $t<0, \mathbf{x} \in \mathbb{R}^{2}$; i.e. $V$ is causal.

(A2) An $R>0$ exists such that $V(t, \cdot)$ is supported in the ball $B(R+t)=\{\mathbf{x}:|\mathbf{x}|<R+t\}$.

(A3) For each $\mathbf{x} \in \mathbb{R}^{2}$ fixed, the function $V(t, \mathbf{x})$ obeys a growth restriction in $t$; we assume boundedness for simplicity, but polynomial growth in time would also be fine.

Assumptions (A1-A3) are common to the whole appendix. Below we also consider an extra assumption on $V$; this is stated later when needed.

Remark C.1. For the target application of Eq. (57), the conditions (A1-A3) on $V=$ $D_{0} W^{+}(\cdot, \cdot, \delta)$ hold, provided there exists a unique, bounded, $C^{1}$ solution $\mathbf{W}$ to the FOSH IBVP (35) corresponding to a sufficiently smooth source $f$ of compact spacetime support.

We aim to show that the formula (86) can be written

$$
\mathcal{I}(t, \mathbf{x} ; V)=\frac{1}{2 \pi} \int_{\mathbb{R}^{2}} d \boldsymbol{\xi} \frac{1}{2 \pi \mathrm{i}} \int_{\eta-\mathrm{i} \infty}^{\eta+\mathrm{i} \infty} d s e^{s t+\mathrm{ix} \cdot \boldsymbol{\xi}} \stackrel{\vee}{P_{1}}(s, \boldsymbol{\xi}) \stackrel{v}{V}(s, \boldsymbol{\xi})
$$

Our argument is classical and assumes $\eta=\operatorname{Re} s>0$. Via a different argument relying on distributions, Ref. [13] make sense of the case $\eta=0$, for which the inversion corresponds to a three-dimensional inverse Fourier transform. Both the argument here and the one in [13] also consider the "forward transform" of $\mathcal{I}(t, \mathbf{x} ; V)$.

C.2. Main results. Our classical argument is based on the following key result.

Lemma C.2. For $\operatorname{Re} s>0, \boldsymbol{\xi} \in \mathbb{R}^{2}$ the expression $M(s, \boldsymbol{\xi})=\stackrel{\vee}{P_{1}}(s, \boldsymbol{\xi})$ in $(84)$ can be written

$$
M(s, \boldsymbol{\xi})=\int_{0}^{\infty} d t e^{-s t} m(t, \boldsymbol{\xi}), \quad m(t, \boldsymbol{\xi})=\frac{1}{\pi} \int_{0}^{1} d \varrho \varrho \int_{0}^{2 \pi} d \theta \exp (\mathrm{i} t \varrho \boldsymbol{\nu}(\theta) \cdot \boldsymbol{\xi}) .
$$

Proof. $M(s, \boldsymbol{\xi})$ is the Laplace transform of $m(t, \boldsymbol{\xi})$, and 29.3.58 of Ref. [19] shows that

$$
m(t, \boldsymbol{\xi})=2(t \xi)^{-1} J_{1}(t \xi) .
$$

Consistently, $M(s, \mathbf{0})=s^{-1}$ corresponds to $m(t, \mathbf{0})=1$, where the latter equation stems from the small- $z$ behavior of $J_{1}(z)$. Now using $\mathbf{1 1 . 3 . 2 0}$ of [19], we write

$$
z^{-1} J_{1}(z)=\int_{0}^{1} d \varrho \varrho J_{0}(\varrho z), \quad m(t, \boldsymbol{\xi})=2 \int_{0}^{1} d \varrho \varrho J_{0}(\varrho \xi t) .
$$

Finally, we get the expression for $m(t, \boldsymbol{\xi})$ in $(90)$ from the identity

$$
J_{0}(z)=\frac{1}{2 \pi} \int_{0}^{2 \pi} d \alpha e^{\mathrm{i} z \cos \alpha}
$$

which in turn follows easily from 9.1.21 of [19]. 
Theorem C.3. With the above assumptions (A1-A3) on $V$ we have that

$$
\mathcal{F}(\mathcal{I}(t, \cdot))(\boldsymbol{\xi})=\mathcal{L}_{\eta}^{-1}(M(\cdot, \boldsymbol{\xi}) \stackrel{\vee}{V}(\cdot, \boldsymbol{\xi}))(t),
$$

where $\mathcal{L}_{\eta}^{-1}$ denotes inverse Laplace transformation using a vertical Bromwich contour crossing the real-axis at position $\eta>0$.

Proof. First, as a consequence of (A1-A2), we have $V(t, \cdot) \in L_{1}\left(\mathbb{R}^{2}\right)$; whence the spatial Fourier transform $\widehat{V}(t, \boldsymbol{\xi}):=\mathcal{F}(V(t, \cdot))(\boldsymbol{\xi})$ exists and is finite. Moreover, by (A1-A3) the Laplace transform of $\widehat{V}(\cdot, \boldsymbol{\xi})$ exists and is finite for Res $>0$. Then by the Laplace convolution theorem,

$$
\mathcal{L}_{\eta}^{-1}(M(\cdot, \boldsymbol{\xi}) \stackrel{v}{V}(\cdot, \boldsymbol{\xi}))=\int_{0}^{t} d t^{\prime} m\left(t-t^{\prime}, \boldsymbol{\xi}\right) \widehat{V}\left(t^{\prime}, \boldsymbol{\xi}\right)
$$

where $m(\cdot, \boldsymbol{\xi})$ is the expression from $(90)$. Therefore,

$$
\mathcal{L}_{\eta}^{-1}(M(\cdot, \boldsymbol{\xi}) \stackrel{\vee}{V}(\cdot, \boldsymbol{\xi}))=\frac{1}{\pi} \int_{0}^{t} d t^{\prime} \int_{0}^{1} d \varrho \varrho \int_{0}^{2 \pi} d \theta \exp \left(\mathrm{i}\left(t-t^{\prime}\right) \varrho \nu(\theta) \cdot \boldsymbol{\xi}\right) \widehat{V}\left(t^{\prime}, \boldsymbol{\xi}\right) .
$$

Notice that

$$
\exp \left(\mathrm{i}\left(t-t^{\prime}\right) \varrho \boldsymbol{\nu}(\theta) \cdot \boldsymbol{\xi}\right) \widehat{V}\left(t^{\prime}, \boldsymbol{\xi}\right)=\frac{1}{2 \pi} \int_{\mathbb{R}^{2}} d \mathbf{x} e^{-\mathrm{ix} \cdot \boldsymbol{\xi}} V\left(t^{\prime}, \mathbf{x}+\left(t-t^{\prime}\right) \varrho \boldsymbol{\nu}(\theta)\right),
$$

i.e. the left-hand side is the Fourier transform of $g(\mathbf{x})=V\left(t^{\prime}, \mathbf{x}+\left(t-t^{\prime}\right) \varrho \boldsymbol{\nu}(\theta)\right)$. Substitution of the last numbered equation into the previous one yields

$$
\mathcal{L}_{\eta}^{-1}(M(\cdot, \boldsymbol{\xi}) \stackrel{\vee}{V}(\cdot, \boldsymbol{\xi}))=\frac{2}{(2 \pi)^{2}} \int_{0}^{t} d t^{\prime} \int_{0}^{1} d \varrho \varrho \int_{0}^{2 \pi} d \theta \int_{\mathbb{R}^{2}} d \mathbf{x} e^{-\mathrm{ix} \cdot \boldsymbol{\xi}} V\left(t^{\prime}, \mathbf{x}+\left(t-t^{\prime}\right) \varrho \boldsymbol{\nu}(\theta)\right) .
$$

For fixed $t$, with $t^{\prime} \in[0, t], \varrho \in[0,1]$, and $\theta \in[0,2 \pi]$, we have assumed that $g(\mathbf{x})=v\left(t^{\prime}, \mathbf{x}+\right.$ $\left.\left(t-t^{\prime}\right) \varrho \boldsymbol{\nu}(\theta)\right)$ is compactly supported in $\mathbf{x}$. Therefore, in the last expression the improper integral over $\mathbb{R}^{2}$ is actually over a finite domain, and therefore also a proper Riemann integral. We may therefore exchange the order of integrations, with the result (98)

$$
\mathcal{L}_{\eta}^{-1}(M(\cdot, \boldsymbol{\xi}) \stackrel{\vee}{V}(\cdot, \boldsymbol{\xi}))=\frac{1}{2 \pi} \int_{\mathbb{R}^{2}} d \mathbf{x} e^{-\mathrm{ix} \cdot \boldsymbol{\xi}}\left[\frac{1}{\pi} \int_{0}^{t} d t^{\prime} \int_{0}^{1} d \varrho \varrho \int_{0}^{2 \pi} d \theta V\left(t^{\prime}, \mathbf{x}+\left(t-t^{\prime}\right) \varrho \boldsymbol{\nu}(\theta)\right)\right] .
$$

We recognize the right-hand side of the last equation as $\widehat{\mathcal{I}}(t, \boldsymbol{\xi}):=\mathcal{F}(\mathcal{I}(t, \cdot))(\boldsymbol{\xi})$.

The last theorem and results from the textbook [20] by Folland then yield the following.

Theorem C.4. $\mathcal{I}(t, \mathbf{x})=\lim _{\varepsilon \rightarrow 0}(2 \pi)^{-1} \int_{\mathbb{R}^{2}} d \boldsymbol{\xi} e^{-\varepsilon^{2} \xi^{2} / 2} e^{\mathrm{ix} \cdot \boldsymbol{\xi}} \mathcal{L}_{\eta}^{-1}(M(\cdot, \boldsymbol{\xi}) \stackrel{v}{V}(\cdot, \boldsymbol{\xi}))(t)$.

Proof. The formula holds since $\mathcal{I}(t, \cdot) \in L_{1}\left(\mathbb{R}^{2}\right)$ by (A1-A2); see page 244 of Ref. [20].

Theorem C.5. Assuming, in addition to (A1-A3), that $V$ is sufficiently regular in space, we have (89). Namely,

$$
\mathcal{I}(t, \mathbf{x})=\mathcal{F}^{-1} \mathcal{L}_{\eta}^{-1}(M(\cdot, \cdot) \stackrel{\vee}{V}(\cdot, \cdot))(t, \mathbf{x}) .
$$


Proof. Due to assumption (A2) on $V$, for each fixed $t$ the function $\mathcal{I}(t, \cdot)$ is also compactly supported in space. Whence $\widehat{\mathcal{I}}(t, \boldsymbol{\xi})$ is analytic in $\boldsymbol{\xi}$. Therefore, provided $\widehat{\mathcal{I}}(t, \boldsymbol{\xi})$ has sufficient decay as $|\boldsymbol{\xi}| \rightarrow \infty$, we have $\widehat{\mathcal{I}}(t, \cdot) \in L_{1}\left(\mathbb{R}^{2}\right)$ and the inversion formula $\mathcal{I}(t, \mathbf{x})=\mathcal{F}^{-1}(\widehat{\mathcal{I}}(t, \cdot))(\mathbf{x})$ follows. Again see page 244 of Ref. [20]. Sufficient spatial regularity for $\mathcal{I}(t, \cdot)$ gives the requisite decay in its spatial Fourier transform. Finally, the spatial regularity of $V(t, \cdot)$ clearly determines the spatial regularity of $\mathcal{I}(t, \cdot)$.

\section{REFERENCES}

[1] G. Bassi, J. A. Ellison, K. Heinemann, and R. Warnock, Microbunching instability in a chicane: Twodimensional mean field treatment, Phys. Rev. ST Accel. Beams 12 (2009) 080704 (24 pages); Transformation of phase space densities under the coordinate changes of accelerator physics, Phys. Rev. ST Accel. Beams 13 (2010) 104403 (11 pages).

[2] G. Bassi, J. A. Ellison, and K. Heinemann, Self Field of Sheet Bunch: A Search for Improved Methods. TH2IOPK01 in ICAP09 Proceedings, San Francisco, California. See JACoW.org.

[3] K. Heinemann, Two topics in particle accelerator beams: Vlasov-Maxwell treatment of coherent synchrotron radiation and topological treatment of spin polarization, Ph.D. dissertation, University of New Mexico, May 2010.

[4] K. Heinemann, D. Bizzozero, J. A. Ellison, and S. R. Lau, Rapid Integration Over History in Self Consistent 2D CSR modeling. TUSDC2 in ICAP12 Proceedings, Rostock-Warnemünde, Germany. See JACoW. org.

[5] T. Hagstrom, T. Warburton, and D. Givoli, Radiation boundary conditions for time-dependent waves based on complete plane wave expansions, J. Comput. Appl. Math., vol. 234, issue 6 (2010) 1988-1995.

[6] T. Hagstrom and T. Warburton, Complete Radiation Boundary Conditions: Minimizing the Long Time Error Growth of Local Methods, SIAM J. Numer. Anal., vol. 47, no. 5 (2009) 36783704.

[7] B. Alpert, L. Greengard, and T. Hagstrom, Rapid evaluation of nonreflecting boundary kernels for time-domain wave propagation, SIAM J. Numer. Anal., vol. 37, no. 4 (2000) 1138-1164.

[8] G. Beylkin and L. Monzon, On approximation of functions by exponential sums, Applied and Computational Harmonic Analysis, 19 (2005) 17-48.

[9] S. Jiang, Fast Evaluation of Nonreflecting Boundary Conditions for the Schrödinger Equation, New York University Ph. D. Dissertation (2001).

[10] K. Xu and S. Jiang, A Bootstrap Method for Sum-of-Poles Approximations, J. Sci. Comput., vol. 55, issue 1 (2013) 16-39.

[11] P. J. Davies and D. B. Duncan, Stability and Convergence of Collocation Schemes for Retarded Potential Integral Equations, SIAM J. Numer. Anal. 42 (2004) 1167-1188

[12] SpEC (Spectral Einstein Code), see www.black-holes.org/SpEC.html.

[13] J. A. Ellison, K. A. Heinemann, and S. R. Lau, Distributional analysis of radiation conditions for the 3+1 wave equation on a half-space, preprint, December 2015.

[14] J. S. Hesthaven, S. Gottlieb, and D. Gottlieb, Spectral Methods for Time-Dependent Problems (Cambridge University Press, Cambridge, 2007).

[15] B. Alpert, L. Greengard, T. Hagstrom, Nonreflecting boundary conditions for the time-dependent wave equation, J. Comput. Phys., vol. 180, issue 1 (2002) 270-296.

[16] S. R. Lau, On Partial Spherical Means Formulas and Radiation Boundary Conditions for the 3+1 Wave Equation, Quart. Appl. Math. 68 (2010) 179-212.

[17] See either www . math .unm. edu/ lau/KernelsRWZ or www .dam.brown. edu/people/sfield/KernelsRWZ.

[18] S. R. Lau, Rapid evaluation of radiation boundary kernels for time-domain wave propagation on blackholes: implementation and numerical tests, Class. Quantum Grav. 21 (2004) 4147-4192.

[19] M. Abramowitz and I. A. Stegun, Handbook of Mathematical Functions (Dover Publishing Inc., New York, 1970).

[20] G. B. Folland, Fourier Analysis and its Applications, (Brooks/Cole Publishing Company, a Division of Wadsworth, Inc., Belmont, 1992). 